\title{
HUBBLE SPACE TELESCOPE AND GROUND-BASED OBSERVATIONS OF THE TYPE Iax SUPERNOVAE SN 2005hk AND SN 2008A
}

\author{
Curtis McCully ${ }^{1}$, Saurabh W. Jha ${ }^{1}$, Ryan J. Foley ${ }^{2,3}$, Ryan Chornock ${ }^{4}$, Jon A. Holtzman ${ }^{5}$, David D. Balam ${ }^{6}$, \\ David Branch ${ }^{7}$, Alexei V. Filippenko ${ }^{8}$, Joshua Frieman ${ }^{9}, 10$, Johan Fynbo $^{11}$, Lluis Galbany ${ }^{12,13}$, \\ Mohan Ganeshalingam ${ }^{8,14}$, Peter M. Garnavich ${ }^{15}$, Melissa L. Graham ${ }^{16,17,18}$, Eric Y. HsiaO ${ }^{18}$, Giorgos Leloudas $^{11,19}$, \\ Douglas C. Leonard ${ }^{20}$, Weidong Li ${ }^{8,29}$, Adam G. Riess ${ }^{21}$, Masao SaKo $^{22}$, Donald P. Schneider ${ }^{23}$, \\ Jeffrey M. Silverman ${ }^{8,24,30}$, Jesper Sollerman ${ }^{11,25}$, Thea N. Steele ${ }^{8}$, Rollin C. Thomas ${ }^{26}$, \\ J. Craig Wheeler ${ }^{24}$, And Chen Zheng ${ }^{27,28}$ \\ ${ }^{1}$ Department of Physics and Astronomy, Rutgers, the State University of New Jersey, 136 Frelinghuysen Road, \\ Piscataway, NJ 08854, USA; cmccully@ physics.rutgers.edu. \\ ${ }^{2}$ Astronomy Department, University of Illinois at Urbana-Champaign, 1002 West Green Street, Urbana, IL 61801, USA \\ ${ }^{3}$ Department of Physics, University of Illinois Urbana-Champaign, 1110 West Green Street, Urbana, IL 61801, USA \\ ${ }^{4}$ Harvard-Smithsonian Center for Astrophysics, 60 Garden Street, Cambridge, MA 02138, USA \\ ${ }^{5}$ Department of Astronomy, MSC 4500, New Mexico State University, P.O. Box 30001, Las Cruces, NM 88003, USA \\ ${ }^{6}$ Dominion Astrophysical Observatory, Herzberg Institute of Astrophysics, 5071 West Saanich Road, Victoria, BC V9E 2E7, Canada \\ ${ }^{7}$ Homer L. Dodge Department of Physics and Astronomy, University of Oklahoma, Norman, OK 73019, USA \\ ${ }^{8}$ Department of Astronomy, University of California, Berkeley, CA 94720-3411, USA \\ ${ }^{9}$ Kavli Institute for Cosmological Physics and Department of Astronomy and Astrophysics, University of Chicago, \\ 5640 South Ellis Avenue, Chicago, IL 60637, USA \\ ${ }^{10}$ Center for Particle Astrophysics, Fermi National Accelerator Laboratory, P.O. Box 500, Batavia, IL 60510, USA \\ ${ }^{11}$ Dark Cosmology Centre, Niels Bohr Institute, University of Copenhagen, Juliane Maries Vej 30, DK-2100 Copenhagen Ø, Denmark \\ ${ }^{12}$ Institut de Física d'Altes Energies, Universitat Autònoma de Barcelona, E-08193 Bellaterra (Barcelona), Spain \\ ${ }^{13}$ Centro Multidisciplinar de Astrofísica, Instituto Superior Técnico, Av. Rovisco Pais 1, 1049-001 Lisbon, Portugal \\ ${ }^{14}$ Lawrence Berkeley National Laboratory, Berkeley, CA 94720, USA \\ ${ }^{15}$ Department of Physics, University of Notre Dame, Notre Dame, IN 46556, USA \\ ${ }^{16}$ Las Cumbres Observatory Global Telescope Network, Goleta, CA 93117, USA \\ ${ }^{17}$ Department of Physics, University of California, Santa Barbara, CA 93106, USA \\ ${ }^{18}$ Carnegie Observatories, Las Campanas Observatory, Colina El Pino, Casilla 601, Chile \\ 19 The Oskar Klein Centre, Department of Physics, Stockholm University, AlbaNova, SE-10691 Stockholm, Sweden \\ ${ }^{20}$ Department of Astronomy, San Diego State University, San Diego, CA 92182, USA \\ ${ }^{21}$ Department of Physics and Astronomy, Johns Hopkins University, Baltimore, MD 21218, USA \\ 22 Department of Physics and Astronomy, University of Pennsylvania, 209 South 33rd Street, Philadelphia, PA 19104, USA \\ ${ }^{23}$ Department of Astronomy and Astrophysics, and Institute for Gravitation and the Cosmos, The Pennsylvania State University, University Park, PA 16802, USA \\ ${ }^{24}$ Department of Astronomy, University of Texas at Austin, Austin, TX 78712, USA \\ ${ }^{25}$ The Oskar Klein Centre, Department of Astronomy, Stockholm University, AlbaNova, SE-10691 Stockholm, Sweden \\ ${ }^{26}$ Computational Cosmology Center, Lawrence Berkeley National Laboratory, 1 Cyclotron Road MS50B-4206, Berkeley, CA, 94720, USA \\ ${ }^{27}$ Kavli Institute for Particle Astrophysics and Cosmology, SLAC National Accelerator Laboratory, 2575 Sand Hill Road, Menlo Park, CA 94025, USA \\ ${ }^{28}$ Department of Physics, Stanford University, Stanford, CA 94305, USA \\ Received 2013 September 17; accepted 2014 March 25; published 2014 April 24
}

\begin{abstract}
We present Hubble Space Telescope (HST) and ground-based optical and near-infrared observations of SN $2005 \mathrm{hk}$ and SN 2008A, typical members of the Type Iax class of supernovae (SNe). Here we focus on late-time observations, where these objects deviate most dramatically from all other SN types. Instead of the dominant nebular emission lines that are observed in other SNe at late phases, spectra of SNe 2005hk and 2008A show lines of Fe II, Ca II, and $\mathrm{Fe}$ I more than a year past maximum light, along with narrow [Fe II] and [Ca II] emission. We use spectral features to constrain the temperature and density of the ejecta, and find high densities at late times, with $n_{e} \gtrsim 10^{9} \mathrm{~cm}^{-3}$. Such high densities should yield enhanced cooling of the ejecta, making these objects good candidates to observe the expected "infrared catastrophe," a generic feature of SN Ia models. However, our HST photometry of SN 2008A does not match the predictions of an infrared catastrophe. Moreover, our HST observations rule out a "complete deflagration" that fully disrupts the white dwarf for these peculiar SNe, showing no evidence for unburned material at late times. Deflagration explosion models that leave behind a bound remnant can match some of the observed properties of SNe Iax, but no published model is consistent with all of our observations of SNe 2005hk and 2008A.
\end{abstract}

Key words: supernovae: general - supernovae: individual (SN 2002cx, SN 2005hk, SN 2008A)

Online-only material: color figures

\section{INTRODUCTION}

The use of Type Ia supernovae (SNe Ia) as distance indicators has revolutionized cosmology with the discovery that the expansion of the universe is currently accelerating, probably

\footnotetext{
${ }^{29}$ Deceased 2011 December 12.

${ }^{30}$ National Science Foundation Astronomy and Astrophysics Postdoctoral Fellow.
}

driven by dark energy (Riess et al. 1998; Perlmutter et al. 1999). Sufficiently large samples have now been collected such that systematic uncertainties are beginning to dominate the statistical uncertainties in SN Ia distances (e.g., Wood-Vasey et al. 2007; Kessler et al. 2009; Conley et al. 2011). Perhaps one of the most fundamental systematic uncertainties stems from the lack of detailed understanding of SN Ia progenitor systems and explosion mechanism. Though exploding white dwarfs typically produce normal SNe Ia (by which we include all objects that fall 
on the one-parameter family correlating luminosity with lightcurve width; Phillips 1993), we are amassing growing evidence that other $\mathrm{SNe}$ are also consistent with a white dwarf origin. Understanding what makes these thermonuclear explosions different can shed light on both normal SNe Ia and more general outcomes of stellar evolution.

SN 2002cx was labeled "the most peculiar known SN Ia" by Li et al. (2003); see also Filippenko (2003). While SN 2002cx was peculiar, it is not unique. SN 2002cx is the prototype for the largest class of peculiar SNe, which we have dubbed "Type Iax" supernovae (for a full description of this class, see Foley et al. 2013). These are weak explosions with luminosities that can fall more than a magnitude below the Phillips relation for normal SNe Ia with similar decline rate, and they have ejecta velocities roughly half those of normal SNe Ia (Jha et al. 2006a). Still, near peak brightness, SNe Iax are similar to $\mathrm{SNe}$ Ia in the general characteristics of their light curves and spectral features. However, the late-time properties of SNe Iax are unmatched by any other previously discovered SN class. Instead of entering a nebular phase dominated by broad forbidden lines of ironpeak elements, the spectrum of SN 2002cx at $\sim 250$ days past maximum brightness ${ }^{31}$ was dominated by permitted $\mathrm{Fe}$ II, with very low expansion velocities $\sim 700 \mathrm{~km} \mathrm{~s}^{-1}$, much lower than ever observed in normal SNe Ia (Jha et al. 2006a). In addition, the late-time spectrum of SN 2002cx showed hints of low-velocity $\mathrm{O}$ I, also unprecedented in SNe Ia, and perhaps an indication of unburned material in the inner regions of the white dwarf.

A variety of models have been proposed to explain the origins of SNe Iax. Branch et al. (2004) and Jha et al. (2006a) suggested that these objects might be explained by pure deflagration models. These models do not explain normal SNe Ia well: a pure deflagration model typically produces much less nickel than is required for the luminosity of a normal SN Ia (Gamezo et al. 2004). Moreover, the highly turbulent and convoluted thermonuclear burning front in these models yields clumpy, well-mixed ejecta, with unburned material, partially burned material, and fully burned (to the iron peak) material at all layers (Röpke 2008), and this mixing is not observed in normal SNe Ia (Gamezo et al. 2004). One of the strongest constraints for a pure-deflagration model is the prediction of unburned material (carbon and oxygen) in the innermost layers, which should be easily detectable in late-time spectra and yet has never been observed in normal SNe Ia (Gamezo et al. 2004).

The problems with the pure deflagration model for normal SNe Ia may become strengths for SNe Iax (Jha et al. 2006a). The low production of nickel and the low luminosity are key traits of SNe Iax. Large amounts of mixing of partially burned, fully burned, and possibly unburned material are observed in all layers of the ejecta. The clumpiness predicted by the pure deflagration model could explain the high densities seen at late times (Jha et al. 2006a; Phillips et al. 2007). In SN 2002cx, there was a tentative detection of O I $\lambda 7774$ (Jha et al. 2006a) and a hint of the line in SN 2005hk, a prototypical SN Iax (Phillips et al. 2007; Stanishev et al. 2007b; Sahu et al. 2008). However, "complete deflagration" models that fully unbind the white dwarf do not predict the high densities at late times seen in SNe Iax, and therefore suggest that oxygen in the inner layers should be revealed by [OI] $\lambda \lambda 6300,6363$ emission (Kozma et al. 2005).

More recently, Jordan et al. (2012) find that if a detonation is not triggered, the explosion is often not powerful enough

\footnotetext{
31 Throughout this paper, $\mathrm{SN}$ phases are given in rest-frame days past $B$-band
} maximum light. to unbind the star, leading to the low luminosities and ejecta velocities like those found in SNe Iax. Kromer et al. (2013) also study the three-dimensional deflagration of a Chandrasekharmass white dwarf. Using radiative transfer models, they find that they can reproduce the luminosity, the early-time light curve, and the early-time spectra of SN 2005hk. Similarly, one of the key features of their explosion simulation is a bound remnant.

The discovery of SN 2008ha sparked controversy about the nature of these peculiar SNe. SN 2008ha was spectroscopically a SN Iax, but it was the most extreme member to date (Foley et al. 2009; Valenti et al. 2009), with maximum-light expansion velocities of just $\sim 2000 \mathrm{~km} \mathrm{~s}^{-1}$, less than half that of even typical SNe Iax. In addition, SN 2008ha was 3 mag fainter than SN 2002cx, with a much more rapid light-curve decline rate. Based on the energetics, and the spectral similarity of SN 2008ha to SN 1997D at late times, Valenti et al. (2009) argued that SN 2008ha was actually a core-collapse SN, rather than a thermonuclear one. Indeed, Moriya et al. (2010) were able to recreate the kinetic energy of SN 2008ha in a corecollapse simulation with large amounts of fallback onto a newly formed black hole (Foley et al. 2009; Valenti et al. 2009). If this model holds for SN 2008ha, Valenti et al. (2009) argued by extension that all SNe Iax might actually be core-collapse $\mathrm{SNe}$. Further support for this idea comes from the fact that, like SN 2008ha, SNe Iax are found almost exclusively in late-type galaxies, similar to core-collapse SNe (Jha et al. 2006a; Foley et al. 2009, 2013). Using $\mathrm{H} \alpha$ maps of a sample of host galaxies of SNe Iax, Lyman et al. (2013) find a statistical association with star forming regions similar to that of SNe IIP. The two objects we focus on in this work, SN 2005hk and SN 2008A, are both in star-forming galaxies, but there is no evidence for star formation at the location of either object (Lyman et al. 2013).

Foley et al. (2010a) published a new set of spectra from earlier epochs of SN 2008ha showing strong evidence for both Si II and $\mathrm{S}$ II at maximum light. While some core-collapse $\mathrm{SNe}$ do show weak Si II lines, the sulfur lines are usually considered hallmarks of thermonuclear burning in a $\mathrm{C} / \mathrm{O}$ white dwarf (sulfur may also be present in the ejecta of other type of SNe, but has never been clearly detected in other type of SNe Foley et al. 2010a); these lines were also seen in SN 2007qd, another SN Iax very similar to SN 2008ha (McClelland et al. 2010). Foley et al. (2010a) proposed that SN 2008ha is better explained by a failed deflagration than a core-collapse model. The host-galaxy distribution is also similar to that of some SNe Ia, specifically SN 1991T-like objects (Foley et al. 2009), and the SN Iax 2008ge exploded in an S0 galaxy with no sign of local star formation to deep limits, inconsistent with a massive star origin (Foley et al. 2010b).

Because these SNe deviate most dramatically from normal $\mathrm{SNe}$ (both core-collapse and thermonuclear) at late times, here we present late-time observations of SNe 2005hk and 2008A (Foley et al. 2013), both typical SNe Iax very similar to $\mathrm{SN} 2002 \mathrm{cx}$, to constrain the nature of this class of SNe. We look for evidence of the [O I ] $\lambda 6300$ line that is predicted for a complete pure deflagration (and is usually seen in core-collapse $\mathrm{SNe}$ ). We also use late-time spectra to infer the composition, velocity structure, density, and temperature of the ejecta.

Besides providing insight to the progenitor systems and explosion mechanisms of white dwarf SNe, SNe 2005hk and 2008A may be ideal candidates to observe the "infrared (IR) catastrophe" predicted by Axelrod (1980), a thermal instability that changes the dominant cooling mechanism from optical lines to far-IR fine-structure lines such as [Fe I] $24 \mu \mathrm{m}$ and [Fe II] 
Table 1

Ground-based Optical Photometry of SN 2005hk from the SDSS-II SN Survey

\begin{tabular}{|c|c|c|c|c|c|c|c|}
\hline $\begin{array}{l}\text { Date } \\
\text { (UT) }\end{array}$ & $\begin{array}{l}\text { MJD } \\
\text { (days) }\end{array}$ & $\begin{array}{l}\text { Phase } \\
\text { (days) }\end{array}$ & $\begin{array}{c}u \\
(\mathrm{mag})\end{array}$ & $\begin{array}{c}g \\
(\mathrm{mag})\end{array}$ & $\begin{array}{c}r \\
(\mathrm{mag})\end{array}$ & $\begin{array}{c}i \\
(\mathrm{mag})\end{array}$ & $\begin{array}{c}z \\
(\mathrm{mag})\end{array}$ \\
\hline 2005 Oct 28 & 53671.34 & -12 & $18.586(0.036)$ & $18.733(0.012)$ & 18.954(0.019) & $19.284(0.027)$ & $19.604(0.104)$ \\
\hline 2005 Nov 2 & 53676.33 & -7 & $16.649(0.031)$ & $16.511(0.004)$ & $16.592(0.004)$ & $16.807(0.005)$ & $17.010(0.011)$ \\
\hline 2005 Nov 5 & 53679.30 & -4 & $16.409(0.031)$ & $16.046(0.009)$ & $16.148(0.009)$ & $16.393(0.015)$ & $16.570(0.014)$ \\
\hline 2005 Nov 7 & 53681.29 & -2 & $16.400(0.031)$ & $15.903(0.004)$ & $15.977(0.003)$ & $16.214(0.005)$ & $16.343(0.009)$ \\
\hline 2005 Nov 23 & 53697.25 & +12 & $18.423(0.031)$ & $16.778(0.015)$ & $15.810(0.006)$ & $15.820(0.006)$ & $15.917(0.016)$ \\
\hline 2005 Nov 26 & 53700.25 & +15 & $18.991(0.038)$ & $17.125(0.022)$ & $15.988(0.013)$ & $15.888(0.014)$ & $15.967(0.018)$ \\
\hline 2005 Dec 01 & 53705.23 & +20 & $19.637(0.050)$ & $17.625(0.013)$ & $16.307(0.003)$ & $16.170(0.008)$ & $16.190(0.009)$ \\
\hline 2006 Aug 28 & 53975.32 & +287 & $\cdots$ & $21.812(0.069)$ & $20.840(0.042)$ & $20.375(0.039)$ & $20.327(0.149)$ \\
\hline 2006 Sep 12 & 53990.34 & +302 & $\cdots$ & $22.051(0.218)$ & 21.194(0.097) & $20.506(0.064)$ & $20.826(0.270)$ \\
\hline 2006 Sep 16 & 53994.35 & +306 & $\cdots$ & $22.242(0.103)$ & $21.121(0.062)$ & $20.528(0.044)$ & $20.666(0.192)$ \\
\hline 2006 Sep 18 & 53996.33 & +308 & $\cdots$ & $22.260(0.127)$ & $21.205(0.064)$ & $20.650(0.062)$ & $20.900(0.301)$ \\
\hline 2006 Sep 27 & 54005.33 & +317 & $\cdots$ & $22.299(0.093)$ & 21.251(0.049) & $20.674(0.042)$ & $20.695(0.162)$ \\
\hline 2006 Sep 30 & 54008.29 & +320 & $\cdots$ & $22.439(0.120)$ & $21.198(0.066)$ & $20.806(0.057)$ & $20.856(0.220)$ \\
\hline 2006 Oct 2 & 54010.28 & +322 & $\cdots$ & $22.441(0.184)$ & $21.225(0.065)$ & $20.723(0.055)$ & $20.615(0.151)$ \\
\hline 2006 Oct 4 & 54012.28 & +324 & $\cdots$ & $22.270(0.315)$ & $21.321(0.157)$ & $20.677(0.070)$ & $20.680(0.207)$ \\
\hline 2006 Oct 12 & 54020.28 & +332 & $\cdots$ & $22.646(0.288)$ & $21.371(0.100)$ & $20.800(0.077)$ & $20.974(0.318)$ \\
\hline 2006 Oct 16 & 54024.36 & +336 & $\cdots$ & $\cdots$ & $21.360(0.098)$ & $21.000(0.092)$ & $\cdots$ \\
\hline 2006 Oct 22 & 54030.28 & +342 & $\cdots$ & $22.533(0.112)$ & $21.466(0.063)$ & $21.017(0.057)$ & $21.230(0.269)$ \\
\hline
\end{tabular}

Note. $1 \sigma$ photometric uncertainties are given in parentheses.

$26 \mu \mathrm{m}$ (Sollerman et al. 2004). This phenomenon has never been observed in normal $\mathrm{SNe}$ Ia even out to 700 days past maximum (Leloudas et al. 2009). Because SNe Iax remain at high densities at late times, the objects in this class should cool faster than normal SNe Ia and should undergo this instability sooner. Using two epochs of late-time HST observations, we compare the color evolution of SN 2008A to the predictions of IR-catastrophe models.

Throughout this paper we adopt $H_{0}=73 \mathrm{~km} \mathrm{~s}^{-1} \mathrm{Mpc}^{-1}$ and correct redshifts $(z)$ using the Virgo+GA infall model of Mould et al. (2000) via $\mathrm{NED}^{32}$ to estimate distances to the SN host galaxies.

\section{OBSERVATIONS AND DATA REDUCTION}

\subsection{Ground-based Optical Photometry and Spectroscopy}

For SN 2005hk, we supplement the ground-based optical photometry of Phillips et al. (2007), Stanishev et al. (2007b), and Sahu et al. (2008) with observations of the equatorial "Stripe 82" from the SDSS-II SN survey (Frieman et al. 2008; Sako et al. 2008), following the reduction procedure detailed by Holtzman et al. (2008). The data comprise ugriz photometry (Fukugita et al. 1996) from the 2005 and 2006 SDSS-II observing seasons, and are presented in Table 1.

The ground-based early-time $B V R I$ observations of SN 2008A were obtained with the $0.76 \mathrm{~m}$ Katzman Automatic Imaging Telescope (Filippenko et al. 2001) and $1 \mathrm{~m}$ Nickel telescope at Lick Observatory (Ganeshalingam et al. 2010). B Vri observations of SN 2008A were also taken as part of the CfA4 survey (Hicken et al. 2012).

Optical spectra of SN 2005hk were obtained with the Low Resolution Imaging Spectrometer (LRIS; Oke et al. 1995) (sometimes in polarimetry mode, LRISp) on the Keck I $10 \mathrm{~m}$ telescope and the Deep Imaging Multi-Object Spectrograph

\footnotetext{
32 http://ned.ipac.caltech.edu
}

(DEIMOS; Faber et al. 2003) on the Keck II 10 m telescope. Spectra of SN 2008A were obtained with the Kast double spectrograph (Miller \& Stone 1993) on the Lick 3 m Shane telescope, Keck I (+ LRIS), and the Dominion Astrophysical Observatory (DAO) Plaskett $1.8 \mathrm{~m}$ telescope. Data-reduction procedures for the Lick and Keck spectra are presented by Silverman et al. (2012); the DAO spectroscopy was reduced with standard techniques. Logs of the observations are provided in Tables 2 and 3, and spectral time series are shown in Figures 1 and 2 . The former figure includes supplementary spectroscopy of SN 2005hk from Chornock et al. (2006), Phillips et al. (2007), Sahu et al. (2008), and Maund et al. (2010). The listed phases are in the $\mathrm{SN}$ rest frame, referenced to $B$ maximum light, which occurred on MJD 53684.2 for SN 2005hk and MJD 54478.3 for SN 2008A.

\subsection{Late-time HST Observations}

Our HST observations of SNe 2005hk and 2008A include optical photometry from WFPC2 and Advanced Camera for Surveys (ACS)/WFC and near-IR photometry from NICMOS and WFC3/IR, which are presented in Table 4. WFPC2 and NICMOS observations of SN 2005hk were taken as part of HST program GO-11133 (PI: Jha). Additional WFPC2 observations of SN 2005hk were available from HST snapshot program GO10877 (PI: Li). SN 2008A was observed in the optical using WFPC2 and ACS and in the near-IR using WFC3/IR as part of HST program GO-11590 (PI: Jha).

The HST observations were combined (with cosmic-ray rejection and subsampling) using MultiDrizzle (Fruchter et al. 2009) with standard parameters. The resulting images of SN 2005hk and SN 2008A are shown in Figures 3-5. We performed aperture photometry on all of the images using the APPHOT task in IRAF. ${ }^{33}$ We used aperture corrections based on encircled

\footnotetext{
33 IRAF is distributed by the National Optical Astronomy Observatory, which is operated by the Association of Universities for Research in Astronomy, Inc. under cooperative agreement with the National Science Foundation.
} 
Table 2

Late-time Spectroscopic Observations of SN 2005hk

\begin{tabular}{lcccccc}
\hline \hline UT & MJD & $\begin{array}{c}\text { Phase } \\
(\text { days })\end{array}$ & Telescope/Instrument & $\begin{array}{c}\text { Exposure } \\
(\mathrm{s})\end{array}$ & $\begin{array}{c}\text { Range } \\
(\AA)\end{array}$ & $\begin{array}{c}\text { Resolution } \\
(\AA)\end{array}$ \\
\hline 2006 Jun 1 & 53887.62 & +201 & Keck I/LRISp & 800 & $5765-7492$ & 3 \\
2006 Jul 1 & 53917.58 & +231 & Keck I/LRISp & 4200 & $3260-9276$ & 7 \\
2006 Nov 23 & 54062.22 & +374 & Keck I/LRIS & 1800 & $3150-9250$ & 7 \\
2006 Dec 23 & 54092.21 & +403 & Keck II/DEIMOS & 1800 & $5000-9300$ & 3 \\
2007 Feb 14 & 54145.23 & +456 & Keck I/LRIS & 1800 & $5500-9240$ & 7 \\
\hline
\end{tabular}

Table 3

Spectroscopic Observations of SN 2008A

\begin{tabular}{lcccccc}
\hline \hline UT & MJD & $\begin{array}{c}\text { Phase } \\
(\text { days })\end{array}$ & Telescope/Instrument & $\begin{array}{c}\text { Exposure } \\
(\mathrm{s})\end{array}$ & $\begin{array}{c}\text { Range } \\
(\AA)\end{array}$ & $\begin{array}{c}\text { Resolution } \\
(\AA)\end{array}$ \\
\hline 2008 Jan 15 & 54480.28 & +2 & Lick/Kast & $1800+1350$ & $3320-10500$ & $6-12$ \\
2008 Jan 25 & 54490.11 & +12 & Plaskett/Cassegrain & 9600 & $3900-7025$ & 6 \\
2008 Feb 12 & 54508.27 & +29 & Keck I/LRIS & 200 & $3075-9340$ & 7 \\
2008 Feb 16 & 54512.23 & +33 & Lick/Kast & 1800 & $3300-10500$ & $6-12$ \\
2008 Aug 3 & 54681.63 & +200 & Keck I/LRIS & $600+300$ & $3270-9196$ & 7 \\
2008 Aug 15 & 54693.18 & +211 & NOT/ALFOSC & 3600 & $4000-9050$ & 8 \\
2008 Aug 28 & 54706.51 & +224 & Keck I/LRIS & 1200 & $3270-9196$ & 7 \\
2008 Oct 27 & 54766.28 & +283 & Keck I/LRIS & 1800 & $3100-9160$ & 7 \\
\hline
\end{tabular}

Table 4

Late-time HST Observations of SN 2005hk and SN 2008A

\begin{tabular}{lcccccc}
\hline \hline Object & UT & MJD & $\begin{array}{c}\text { Phase } \\
\text { (days) }\end{array}$ & Instrument/Filter & $\begin{array}{c}\text { Exposure } \\
(\mathrm{s})\end{array}$ & $\begin{array}{c}\text { Magnitude } \\
(\mathrm{mag})\end{array}$ \\
\hline SN 2005hk & 2007 May 31 & 54251.11 & +560 & WFPC2/F450W & 1600 & $>25.50$ \\
SN 2005hk & 2007 May 31 & 54251.12 & +560 & WFPC2/F675W & 900 & $>24.80$ \\
SN 2005hk & 2007 May 31 & 54251.91 & +561 & WFPC2/F555W & 920 & $>25.50$ \\
SN 2005hk & 2007 May 31 & 54251.92 & +561 & WFPC2/F814W & 1400 & $24.91(0.22)$ \\
SN 2005hk & 2007 Jun 25 & 54276.09 & +585 & NIC2/F110W & 5120 & $>25.86$ \\
SN 2005hk & 2007 Jun 27 & 54278.68 & +588 & WFPC2/F555W & 460 & $>25.20$ \\
SN 2005hk & 2007 Jun 27 & 54278.69 & +588 & WFPC2/F814W & 700 & $>24.55$ \\
SN 2005hk & 2007 Aug 13 & 54325.45 & +634 & WFPC2/F606W & 4800 & $26.35(0.15)$ \\
SN 2005hk & 2007 Aug 13 & 54325.91 & +634 & WFPC2/F814W & 1600 & $>25.10$ \\
SN 2005hk & 2007 Aug 15 & 54327.24 & +636 & NIC2/F160W & 7680 & $>25.08$ \\
SN 2008A & 2009 Feb 19 & 54881.15 & +396 & WFPC2/F555W & 1000 & $24.20(0.11)$ \\
SN 2008A & 2009 Feb 19 & 54881.17 & +396 & WFPC2/F791W & 900 & $23.10(0.10)$ \\
SN 2008A & 2009 Feb 19 & 54881.21 & +396 & WFPC2/F622W & 800 & $24.14(0.13)$ \\
SN 2008A & 2009 Feb 19 & 54881.24 & +396 & WFPC2/F850LP & 1450 & $23.40(0.26)$ \\
SN 2008A & 2009 Feb 20 & 54882.88 & +397 & WFPC2/F439W & 1500 & $>24.35$ \\
SN 2008A & 2009 Aug 18 & 55061.34 & +573 & ACS/F625W & 3530 & $26.15(0.06)$ \\
SN 2008A & 2009 Aug 18 & 55061.41 & +573 & ACS/F555W & 3750 & $26.25(0.05)$ \\
SN 2008A & 2009 Aug 18 & 55061.54 & +573 & ACS/F775W & 2484 & $26.25(0.11)$ \\
SN 2008A & 2009 Aug 18 & 55061.61 & +573 & WFC3IR/F110W & 8335 & $26.00(0.21)$ \\
\hline
\end{tabular}

Note. $1 \sigma$ photometric uncertainties are given in parentheses. Upper limits are $3 \sigma$.

energies given by Holtzman et al. (1995) for WFPC2, Sirianni et al. (2005) for ACS/WFC, and the WFC3 Instrument handbook (Wong et al. 2010) for WFC3/IR. For NICMOS, we generated a point-spread function model using the TinyTim software (Krist 1993, but see Hook \& Stoehr 2008) and then directly measured encircled energies from the model. For the ACS and WFPC2 data we also corrected for the charge-transfer inefficiency of the detectors, using the models of Dolphin (2009) for WFPC2 and Chiaberge et al. (2009) for ACS/WFC. To derive upper limits in cases without a significant detection of the SN, we injected fake stars with a range of magnitudes into the images. These artificial stars were photometered in the same way as the SNe, with the standard deviation of the recovered magnitudes used to estimate the photometric uncertainty. We list our derived photometry and $3 \sigma$ upper limits in Table 4.
Both SNe 2005hk and 2008A are typical members of the SN Iax class. These two SNe along with SN 2002cx are quite homogeneous in both their light curves and their spectra. Figure 6 shows the spectroscopic similarity of SNe 2002cx, 2005hk, and 2008A. In Figure 7, we plot photometry for SNe 2002cx (Li et al. 2003; Jha et al. 2006a), 2005hk (Phillips et al. 2007; Sahu et al. 2008, and this work), and 2008A, compared to the normal SN Ia 1992A (Kirshner et al. 1993), all extending to late times.

\section{RESULTS}

\subsection{Lack of Near-infrared Secondary Maxima in SNe Iax}

At early times, SN 2008A mimics the photometric behavior shown by SN 2002cx and SN 2005hk, with broader light curves 


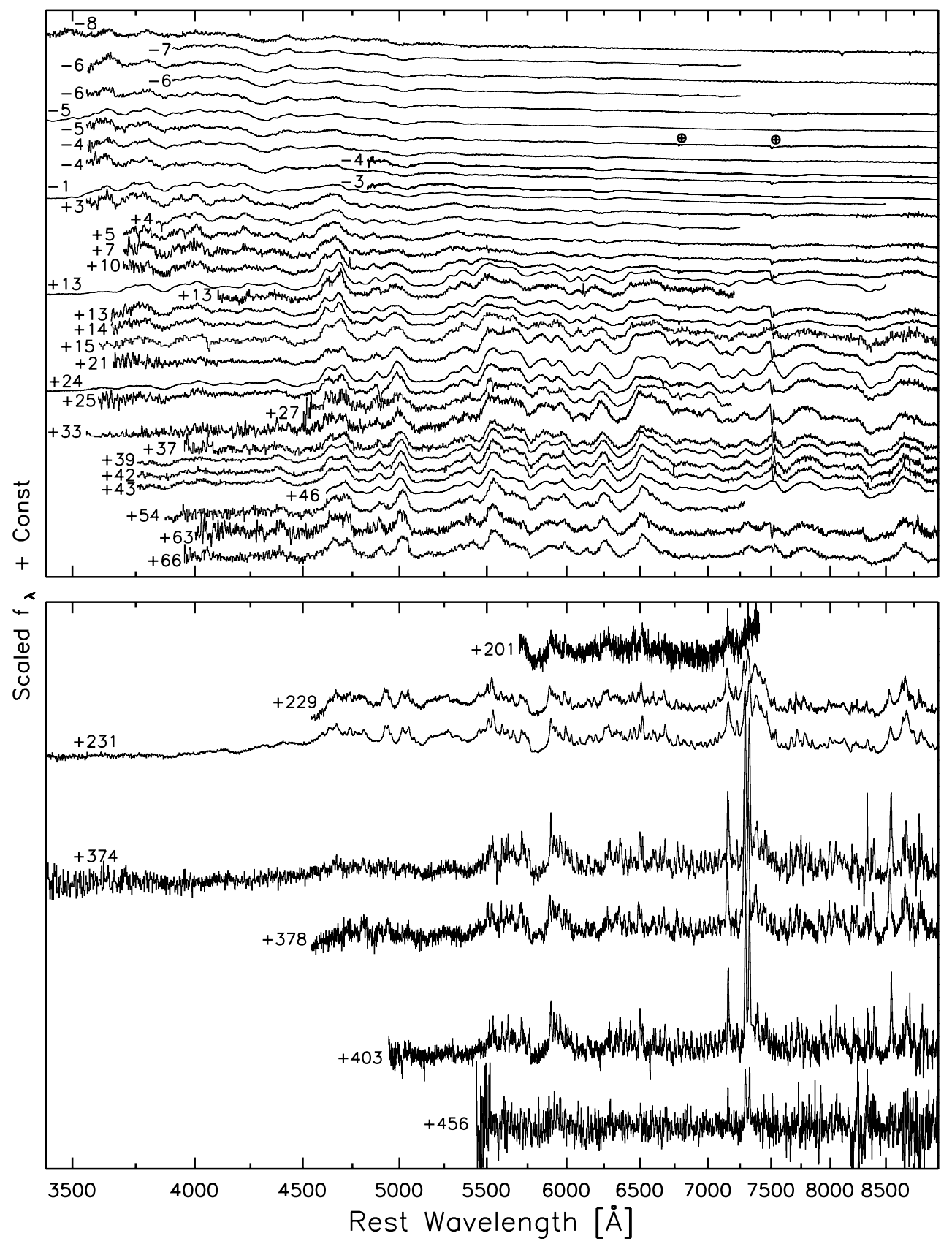

Figure 1. Spectral time series of SN 2005hk, labeled by phase (in days). Both the phases and wavelengths are corrected to the SN rest frame using $z=0.01176$ from the [Ca II] $\lambda \lambda 7291,7323$ lines. We include spectra from Chornock et al. (2006), Phillips et al. (2007), Sahu et al. (2008), and Silverman et al. (2012). The top panel shows the early-time spectra and the bottom panel shows the late-time spectra.

than SN 1992A, particularly in the redder bands, as shown in the insets of Figure 7. These SNe Iax do not show a second maximum in the near-infrared ( $\mathrm{Li}$ et al. 2003; Phillips et al. 2007). This is puzzling if the secondary maximum is ascribed to a transition in the dominant ionization state (Fe III to Fe II; Kasen 2006), because SNe Iax have hot early-time spectra with prominent Fe III, similar to SN 1991T-like SNe Ia that do show a strong secondary maximum. Low-luminosity SNe Ia like SN 1991bg lack secondary maxima (Filippenko et al. 1992a; Leibundgut et al. 1993); in the ionization model this is explained by an earlier transition onset in the cooler spectra, merging the primary and secondary maxima. SNe Iax could undergo much more rapid cooling near maximum light than normal SNe Ia (to explain the Fe III in the spectrum and yet lack of a secondary maximum). This is plausible as SNe Iax remain at high densities at late times instead of entering a nebular phase, which should enhance the cooling. However, we do not see evidence for rapid cooling in our late-time observations, so perhaps an alternate model is needed.

\subsection{Spectral Features and Velocity Structure}

One of the defining characteristics of SNe Iax is the low velocity of their spectral features. At early times, typical $\mathrm{SNe}$ Iax have expansion velocities for features such as $\mathrm{Si}$ II of $\lesssim 5000 \mathrm{~km} \mathrm{~s}^{-1}$, roughly half those seen in normal SNe Ia (Foley et al. 2013), though the overall appearance of the spectra is otherwise similar to those of SN 1991T-like and SN 1999aa-like SNe Ia. SNe Iax at late times are dominated by permitted lines from iron-group elements rather than entering 


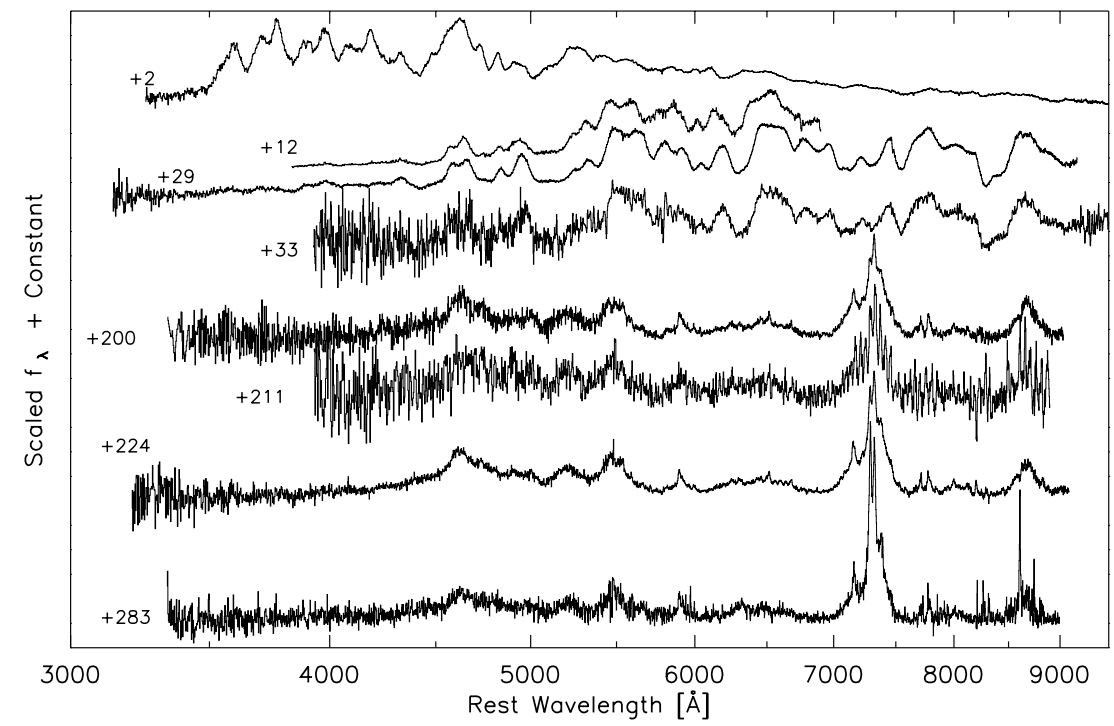

Figure 2. Spectral time series of SN 2008A, labeled by phase (in days). Both the phases and wavelengths are corrected to the SN rest frame using $z=0.01825$ determined from the $[\mathrm{Ca}$ II $] \lambda \lambda 7291,7323$ lines.

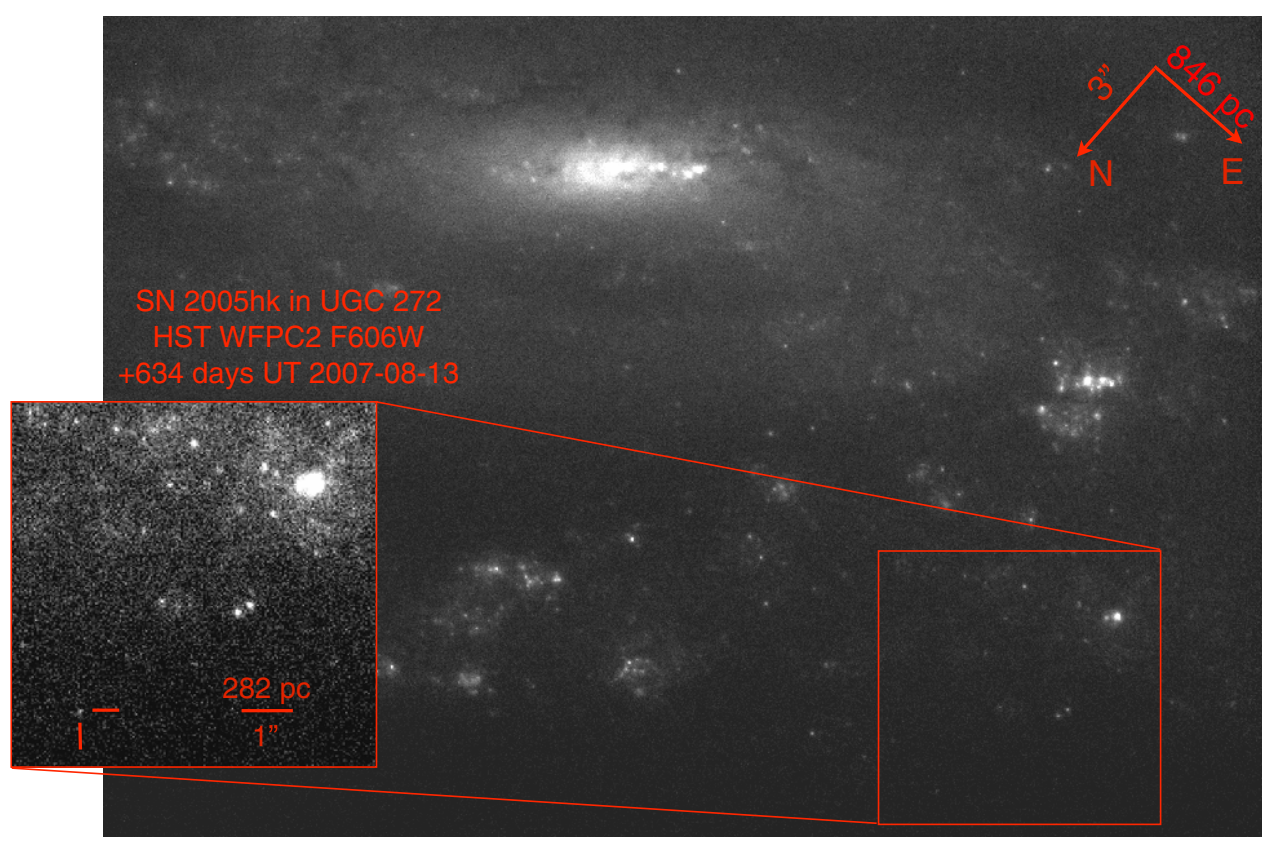

Figure 3. F606W observations of SN 2005hk in UGC $272(z=0.013)$ using the WFPC 2 instrument on $H S T$ taken 634 days after $B$ maximum. The SN is marked in the inset, which has been rescaled to better show the SN.

(A color version of this figure is available in the online journal.)

a nebular phase like normal SNe Ia (Jha et al. 2006a; Foley et al. 2013). Nonetheless, a few forbidden lines are present, and the strongest features in late-time SN Iax spectra are the $[\mathrm{Ca}$ II $] \lambda \lambda 7291,7323$ doublet and [Fe II] $\lambda 7155$ (Jha et al. 2006a; Sahu et al. 2008; Foley et al. 2013). Using these features, we derive the redshift of the ejecta of SNe 2002cx, 2005hk, and 2008 A to be $z=0.02323,0.01176$, and 0.01825 , respectively. Ideally, we would use the redshift of the progenitor system, but this is not possible, so we adopt these redshifts to compare the shape of spectral features between objects. These emission lines have unprecedentedly low velocities: in the last observed epoch, SN 2005hk had velocities with an FWHM as small as $\lesssim 500 \mathrm{~km} \mathrm{~s}^{-1}$, the lowest ever measured for any white-dwarf SN (SN Ia or SN Iax; Phillips et al. 2007). Some faint SNe IIP also have low velocities, within a factor of two of SN $2005 \mathrm{hk}$ at similar phase, but these typically do not get to a FWHM of $500 \mathrm{~km} \mathrm{~s}^{-1}$ until $\sim 600$ days past maximum (Maguire et al. 2012).

Figures 1 and 2 show the full spectroscopic evolution of SN 2005hk and SN 2008A, respectively. The features of SN 2005hk and SN 2008A match well at all epochs as illustrated in Figure 6. We used Synapps (an optimizer for Syn++ Thomas et al. 2011b), based on the SYNOW code (Branch et al. 2005), to identify the strongest features in the photospheric spectra of SN 2005hk and SN 2008A and is shown in Figure 8. Both objects are dominated by iron group elements, including Fe III emission similar to 91T-like spectra but with significant contributions from intermediate-mass elements. We also detect (presumably) unburned material in both objects: both SN 2005hk and SN 2008A have emission from O I and 

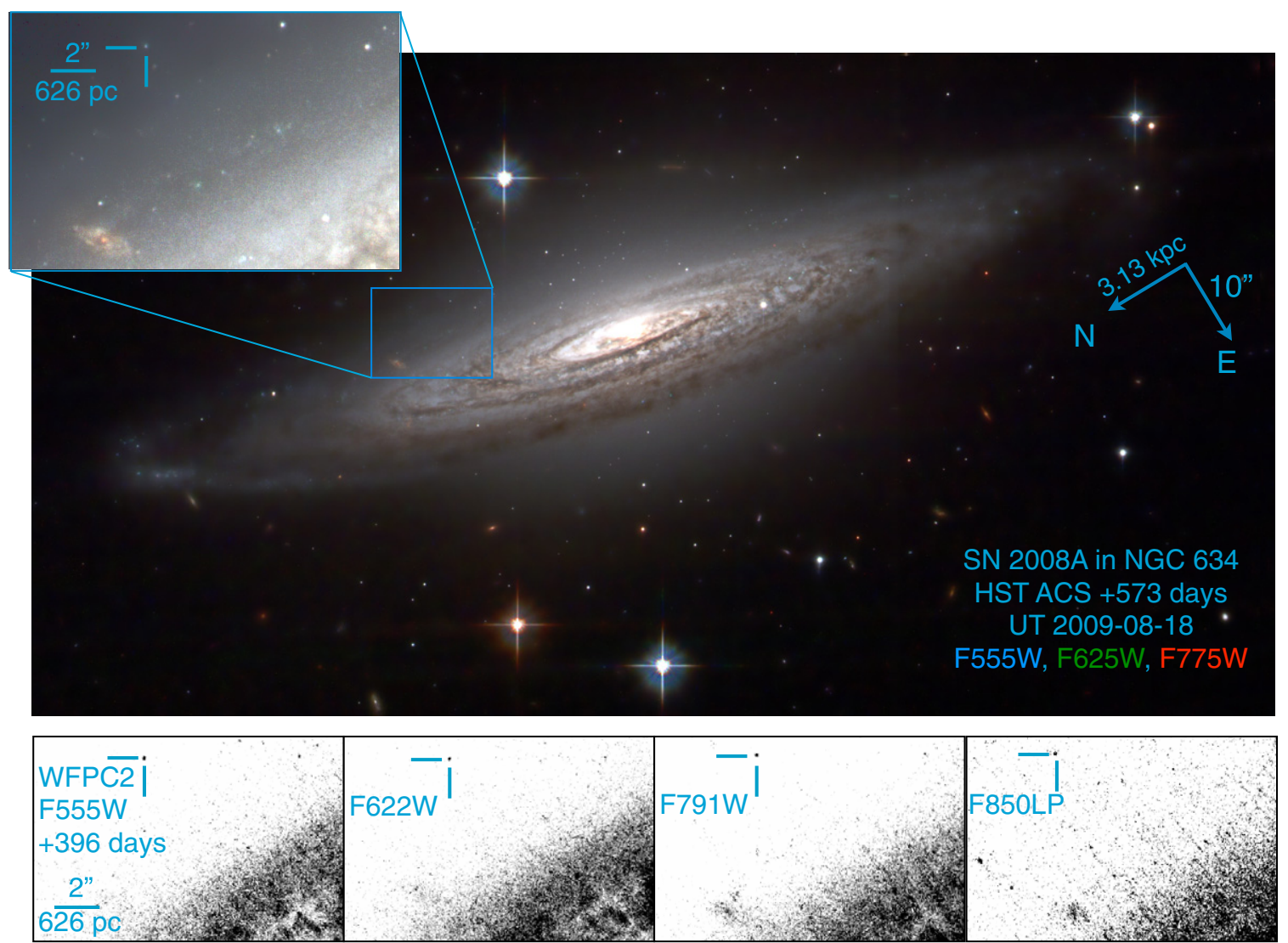

Figure 4. $H S T$ observations of SN 2008A in NGC $634(z=0.016)$. The top image is combined from Vri (F555W, F625W, F775W) data taken with ACS, 573 days after maximum light. SN 2008A is marked in the inset image, in the outskirts of its host. Color image was produced using STIFF (Bertin 2012). An unsharp mask filter has been applied to the color figure to emphasize faint sources for display purposes. The bottom panels show WFPC2 observations of SN 2008 A taken 396 days after maximum light, in the F555W, F622W, F791W, and F850LP filters. Like SN 2005hk, SN 2008A is located in the outskirts of a spiral galaxy.

(A color version of this figure is available in the online journal.)

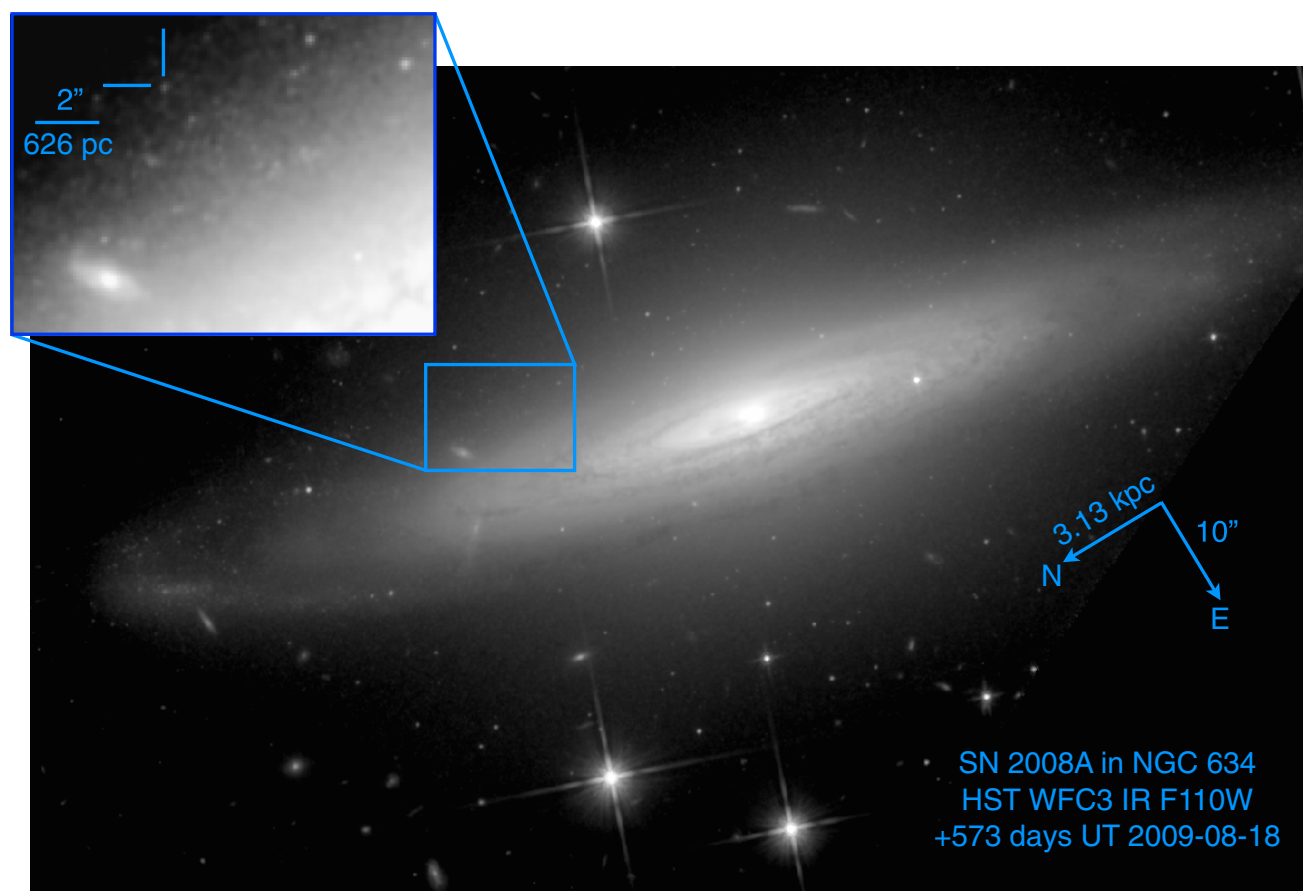

Figure 5. HST near-infrared observations of SN 2008A in NGC 634, taken with WFC3/IR in the F110W passband, 573 days after maximum light. (A color version of this figure is available in the online journal.) 


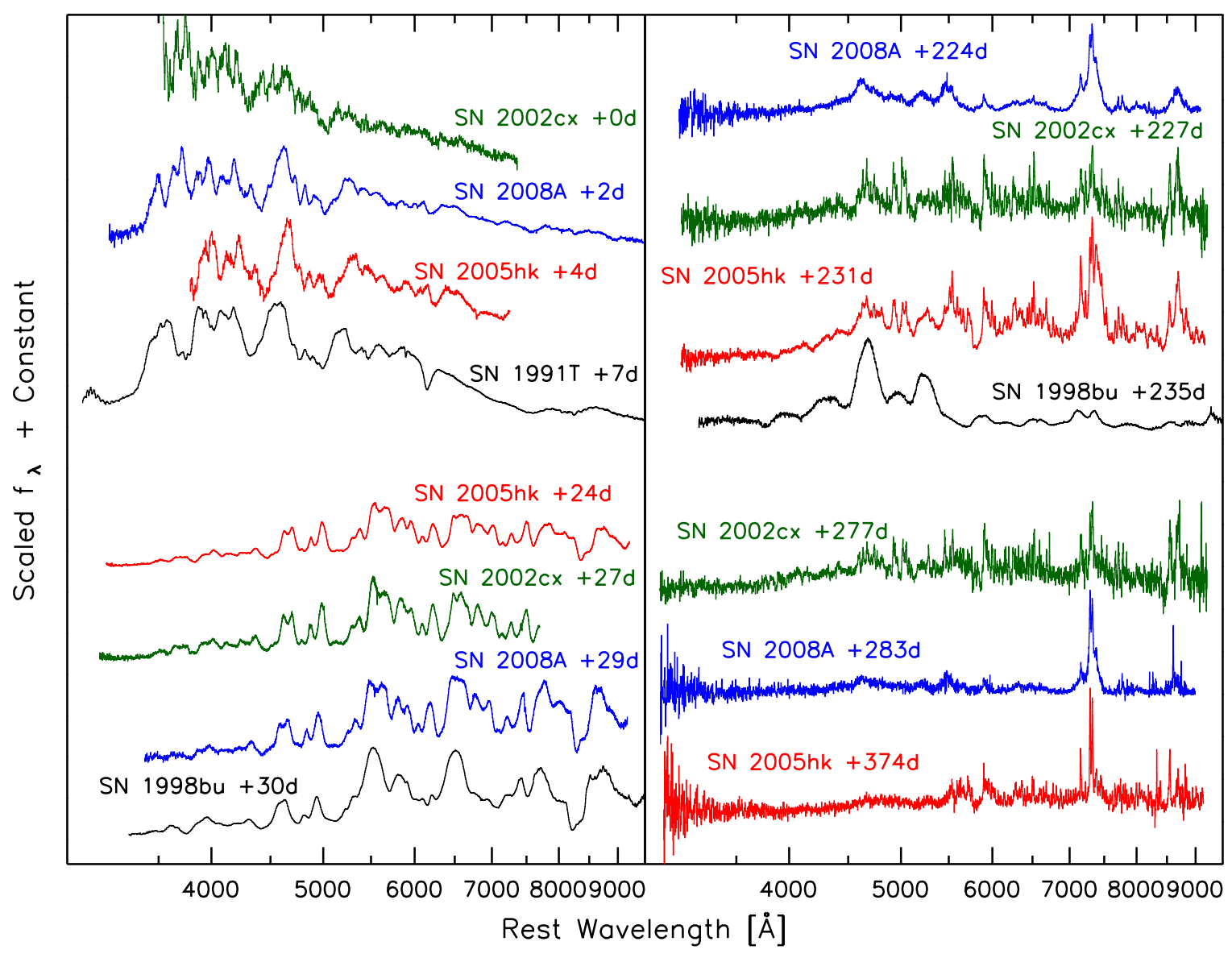

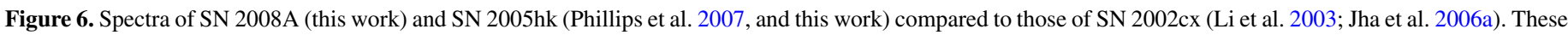

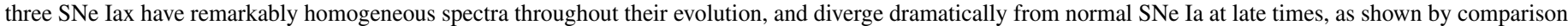
spectra of SN 1991T (Filippenko et al. 1992b) and SN 1998bu (Jha et al. 1999; Li et al. 2001; Silverman et al. 2012).

(A color version of this figure is available in the online journal.)

possibly O II. SN 2008A has strong carbon features (even relative to normal SNe Ia), but SN 2005hk shows no evidence for carbon emission. One of the key differences between these two objects is that the velocities of the spectral features of SN 2008A are higher by $1500 \mathrm{~km} \mathrm{~s}^{-1}$ at maximum than those of SN 2005hk. The spectral features of SN 2005hk remain at lower velocities that those of SN 2008A at all epochs, even out to a year past maximum.

Figure 9 compares the late-time spectra of SN 2002cx, SN 2005hk, and SN 2008A. While these spectra are qualitatively similar, there are key differences (also analyzed in detail by Foley et al. 2013). At all epochs the velocities of SN 2008A are higher than those of SN 2005hk, similarly to the photospheric spectra. SN 2002cx shows a stronger Ca near-IR triplet than either SN 2005hk or SN 2008A. In the spectra of SN 2008A, [Fe II] $\lambda 7155$ has an asymmetric profile. This may be caused by contamination from another line; there is a [CoI] feature at the wavelength in question, but no other [Co I] lines are seen (including lines more easily excited), making this option unlikely.

Sahu et al. (2008) identify [Fe II] $\lambda 7389$ in their spectra of SN 2005hk. However, there is another strong feature, [Ni II] $\lambda 7378$, at almost the same wavelength. The feature near these wavelengths is broader than the [Fe II] $\lambda 7155$ line, so we argue that it is likely a blend of these two lines. There is a strong line that is near [Fe II] $\lambda 8617$ in SN 2008A that is not seen clearly in $\mathrm{SNe} 2002 \mathrm{cx}$ or $2005 \mathrm{hk}$, but is observed in the normal SN Ia
$2003 \mathrm{hv}$ and has been used to measure asymmetry in the inner layers of the ejecta (Leloudas et al. 2009).

We used Syn++ (Thomas et al. 2011b) to model the permitted lines in the late-time spectra. Our results are also shown in Figure 9. Because the signal-to-noise ratio was the highest in the latest SN 2005hk spectrum, we fit the lines in this spectra and then matched them to the spectra of SNe 2002cx and 2008A. We find that $\mathrm{Fe}$ II is necessary to fit many of the lines between 6000 and $6400 \AA$, as was found by Jha et al. (2006a) and confirmed by Sahu et al. (2008). While many lines are fit well with Fe II, some had remained unidentified. We find that Fe I significantly improves the fit of the late-time SN 2002cx and SN 2005hk spectra. There are strong lines to the red of the P Cygni profile of Na I $\lambda 5891$ that can be seen in all three objects (SNe 2002cx, 2005hk, and 2008A) that are well fit by Fe I with an excitation temperature near $5000 \mathrm{~K}$. However, there are also [Fe I] lines that match these features, making the identification of these lines ambiguous.

The velocity structure of the forbidden lines relative to the host galaxy is interesting. Figure 10 displays the [Ca II] $\lambda \lambda 7291,7323$ doublet and [Fe II] $\lambda 7155$ line referenced to the host-galaxy (nucleus) rest frame (as opposed the rest frame of the ejecta used elsewhere in this work). As the figure shows, the line velocities are largely consistent over time, though the line widths decrease as the SNe evolve. Foley et al. (2013) found that the $[\mathrm{Ca} \mathrm{II}]$ and $[\mathrm{Fe} \mathrm{II}]$ features were shifted in opposite directions relative to the host rest frame for the majority of SNe Iax, but for 

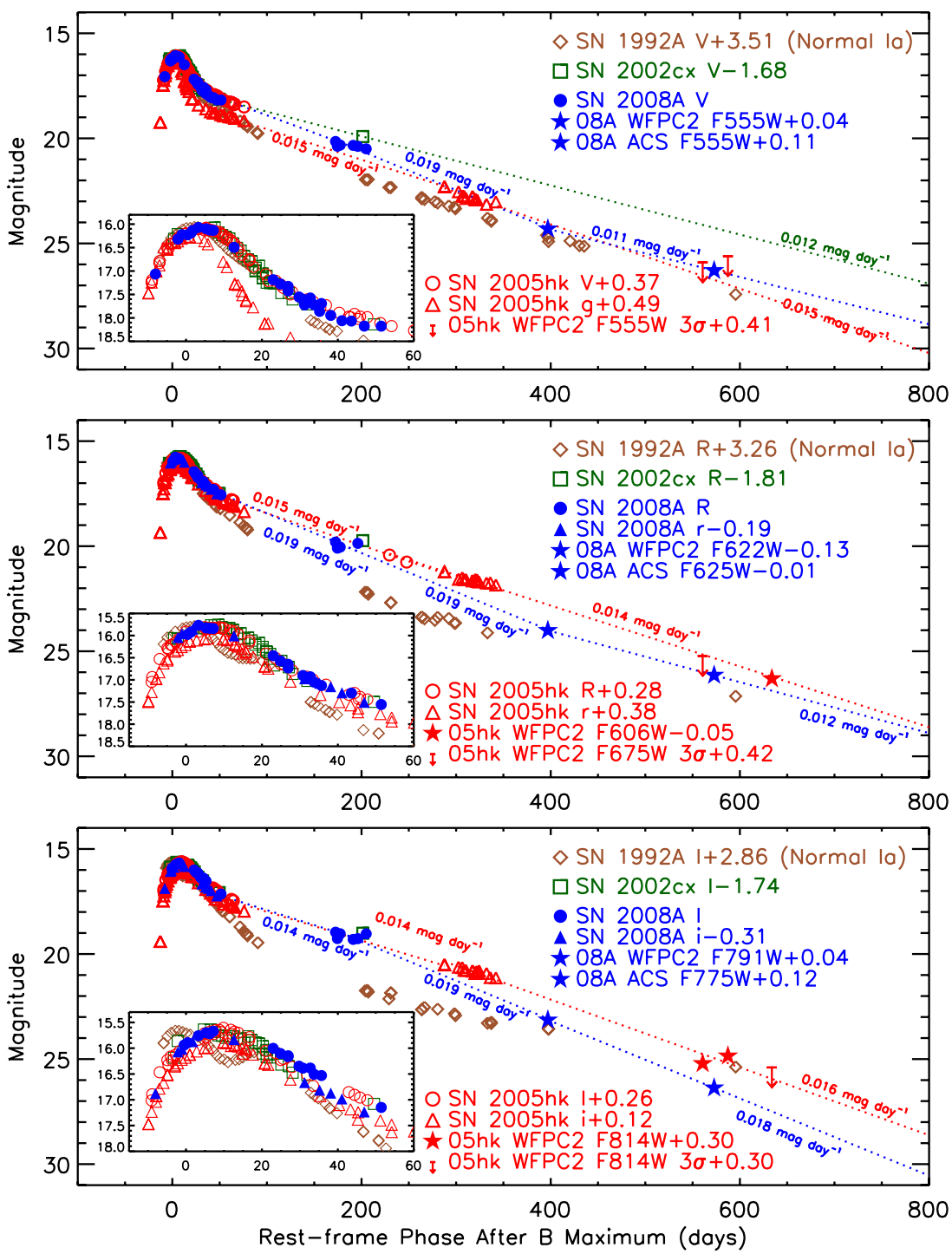

Figure 7. Light curves of SN 2002cx (Li et al. 2003; Jha et al. 2006a), SN 2005hk (Phillips et al. 2007; Sahu et al. 2008, and this work), and SN 2008A (Ganeshalingam et al. 2010; Hicken et al. 2012, and this work), compared to those of the normal Type Ia SN 1992A (Kirshner et al. 1993). These include both ground-based and HST observations as noted. The light curves have been shifted to match the peak of SN 2008A in $V, R$, and $I$. In each of the optical bands the decline rate is faster than the predicted 0.0098 mag day ${ }^{-1}$ for the decay of ${ }^{56} \mathrm{Co}$.

(A color version of this figure is available in the online journal.)

these three objects (also part of the Foley et al. 2013 sample), we do not confirm this pattern, and find consistent velocities from both [Ca II] and [Fe II].

In SN 2002cx, these lines were blueshifted by $220 \pm 52 \mathrm{~km} \mathrm{~s}^{-1}$ relative to the SN host galaxy. In SN 2005hk, the lines were also blueshifted by $370 \pm 22 \mathrm{~km} \mathrm{~s}^{-1}$. Contrarily, in SN 2008A the lines were redshifted by $547 \pm 10 \mathrm{~km} \mathrm{~s}^{-1}$. These velocity shifts are significantly in excess of the host-galaxy rotation speeds, which have maximum rotation velocities of $128 \pm 14 \mathrm{~km} \mathrm{~s}^{-1}$ (CGCG 044-035, host of SN 2002cx), 108 $\pm 4 \mathrm{~km} \mathrm{~s}^{-1}$ (UGC 272, host of SN 2005hk), and $225 \pm 7 \mathrm{~km} \mathrm{~s}^{-1}$ (NGC 634, host of SN 2008A), respectively (Paturel et al. 2003). This implies that the velocity shifts are intrinsic to the $\mathrm{SN}$ explosion. We discuss the interpretation of these results in Section 4.

\subsection{Temperature and Density}

Important constraints for models are provided by the physical properties of the ejecta, specifically the temperature and density at late epochs. As discussed above, the late-time spectra of
SNe Iax are dominated by permitted iron transitions which would imply that the electron density has remained higher than in normal SNe Ia. More quantitatively, the ratio of the [Ca II] doublet $(\lambda \lambda 7291,7323)$ to the permitted Ca II near-IR triplet can be used to constrain the electron density and temperature (Ferland \& Persson 1989). As there is a degeneracy between temperature and density in this method, to infer the electron density we need an independent method for constraining the allowed temperatures.

The presence of both Fe I and Fe II transitions in the spectra of SN 2005hk is only allowed for a narrow range of temperatures, if these lines arise from the same regions in the ejecta, as suggested by the similar line profiles and velocities. The Saha equation predicts a transition between Fe II and Fe I transitions at $\sim 4500 \mathrm{~K}$, and in local thermodynamic equilibrium (LTE) calculations, Hatano et al. (1999) find optical depth exceeding unity in both Fe II and Fe I for $T \lesssim 7000 \mathrm{~K}$. Although we do not expect equilibrium conditions, and non-LTE effects are likely to be important, we can still use these estimates as a reasonable range for the ejecta temperature, based on the presence and 


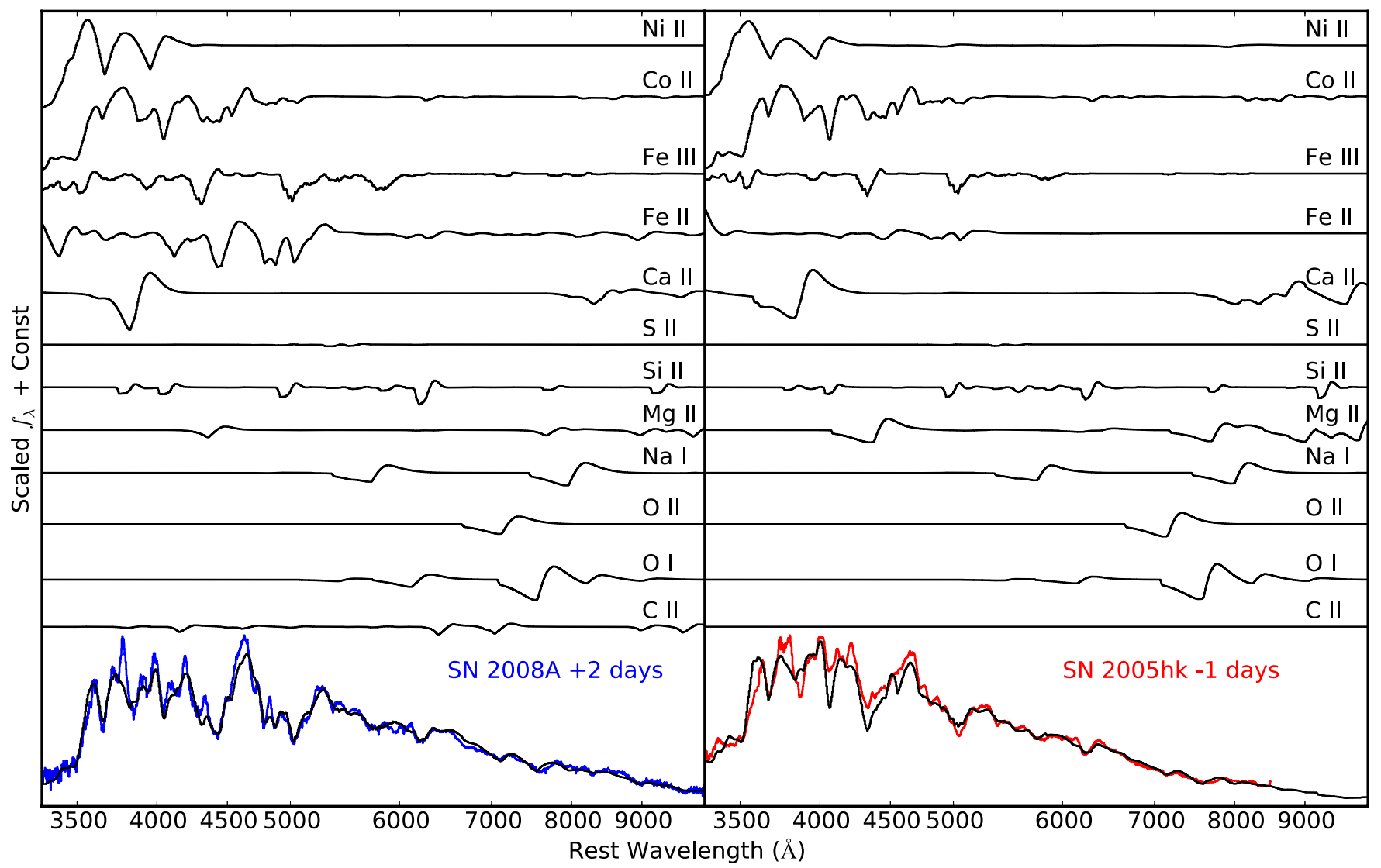

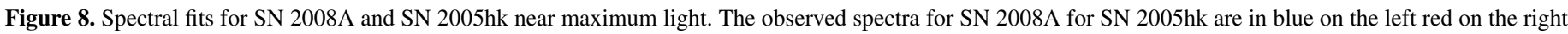

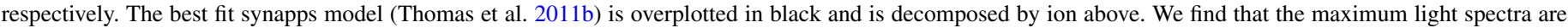

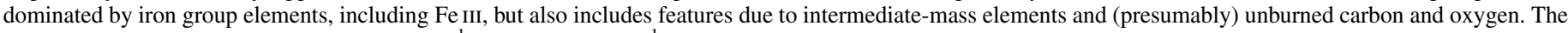
best-fit velocity for SN $2005 \mathrm{hk}$ is $\sim 7000 \mathrm{~km} \mathrm{~s}^{-1}$, about $1500 \mathrm{~km} \mathrm{~s}^{-1}$ lower than SN 2008A. This velocity difference persists at all epochs.

(A color version of this figure is available in the online journal.)

strength of the $\mathrm{Fe}$ I features among numerous permitted lines with similar P Cygni line profiles and velocity structure. In Figure 11, we show the inferred densities in SN 2002cx, SN 2005hk, and SN 2008A derived from the Ca II flux ratio for our allowed range of temperatures. We are assuming that the iron and calcium are microscopically mixed; this is plausible given their overlapping velocity ranges, but we cannot definitively say they reside in the same physical region.

The spectra of all three of our objects are consistent with little or no density evolution, and all have electron densities $\sim 10^{9} \mathrm{~cm}^{-3}$. Free expansion would predict that the density should go as $t^{-3}$, but this is in clear contradiction with the observations. Our measurements of the density are marginally consistent with $t^{-1}$, but only if the temperature decreases slowly. If the temperature decreases more rapidly (as might be expected given the high densities, if radiative cooling dominates), the density is likely evolving even more slowly than $t^{-1}$, remaining roughly constant, or perhaps even increasing. One possible explanation, given the decreasing line widths we observe (Figure 10), is that we are continuing to see the "photosphere" recede to ever lower velocities, and for some reason the emitting region has a roughly constant density as the ejecta dilute.

\subsection{Oxygen at Late Times}

As described in Section 1, pure deflagration models have been raised as a possibility to explain SN Iax explosions. These models generically predict a turbulent burning front which causes strong mixing in all layers of the ejecta, implying there should be unburned material (presumably $\mathrm{C}$ and $\mathrm{O}$ ) in the innermost layers of the ejecta (Gamezo et al. 2003). At late epochs, one of the strongest spectral features should be [O I] $\lambda 6300$ (assuming the entire white dwarf is disrupted). (Kozma et al. 2005).

There is no evidence of this line in the late-time nebular spectra of normal SNe Ia. ${ }^{34}$ Instead, the nebular spectra of normal SNe Ia are dominated by forbidden transitions of ironpeak elements. The favored explanation for this is that the burning front transitioned from a subsonic deflagration to a supersonic detonation (Khokhlov 1991; Gamezo et al. 2005), which subsequently burns the central material uniformly to the iron peak.

SNe Iax can have strong carbon features before and at maximum light (like SN 2008A as discussed above), corresponding to unburned material in the outer layers of the ejecta (Foley et al. 2013, and references therein). Some normal SNe Ia also show these lines at early times, but if present, they usually disappear by maximum light (Thomas et al. 2007, 2011a; Parrent et al. 2011; Folatelli et al. 2012; Silverman \& Filippenko 2012). This may imply that unburned material is present at higher mass fractions deeper into the ejecta in SNe Iax compared to normal $\mathrm{SNe}$ Ia. If SNe Iax are to be explained as pure deflagrations (no transition to supersonic burning), we expect to see evidence of

\footnotetext{
34 Taubenberger et al. (2013) have recently shown the evidence for nebular [O I] $\lambda \lambda 6300,6363$ with a complex line provide in a late-time spectrum of SN 2010lp, a subluminous SN 1991bg-like supernova.
} 


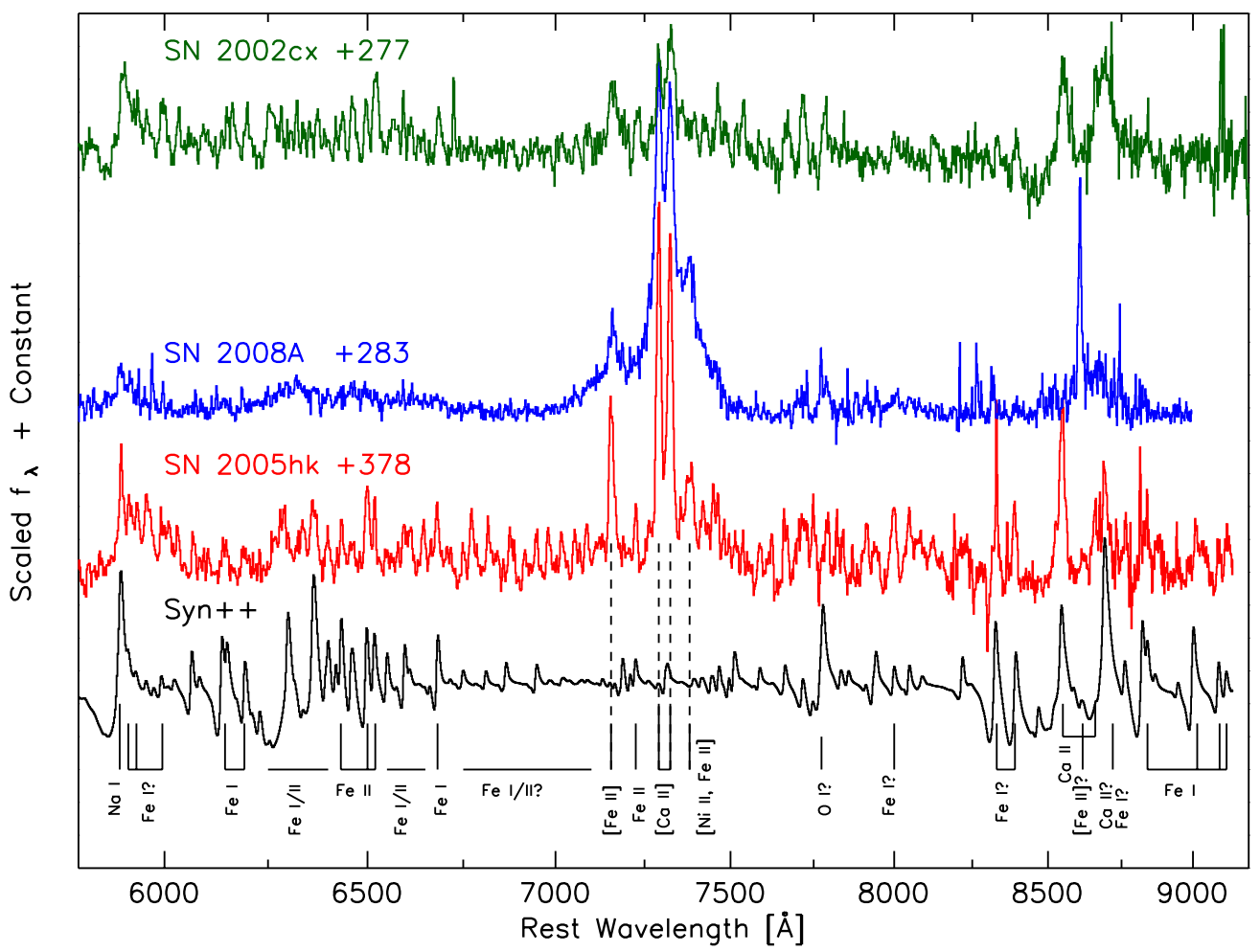

Figure 9. Late-time spectra of SN 2002cx ( + 277 days; top, green), SN 2008A (+ 283 days; top middle, blue), and SN $2005 \mathrm{hk}$ ( +378 days; bottom middle, red; Sahu et al. 2008) compared to a synthetic model spectrum (black, bottom). The synthetic spectrum was created with Syn++ (Thomas et al. 2011b, similar to SYNOW (Branch et al. 2005)). The synthetic spectrum assumes Boltzmann excitation and only models the permitted lines. Non-LTE effects and forbidden lines are important for the relative strengths of the lines, but Syn++ is useful for the identification of the lines. We have marked the strongest forbidden emission features, discussed in the text, with dashed lines. The signal-to-noise ratio is substantially better in SN 2005hk, so this was used for the primary fit and then compared to SN 2002cx and SN 2008A. Line identifications are included under the synthetic spectrum.

(A color version of this figure is available in the online journal.)

unburned material at all velocities, including the central regions that are revealed at late times.

Jha et al. (2006a) tentatively identified permitted O I $\lambda 7774$ in SN 2002 cx at 227 and 277 days after $B$ maximum. As can be seen in Figure 9, the same feature is present in SN 2008A, but even stronger. If the identification is correct, this matches the model predictions of a pure deflagration explosion that never transitioned to a detonation. However, this feature is a permitted transition, implying that the density of the ejecta is unexpectedly high out to $\sim 280$ days past maximum. By this phase, SNe Ia have transitioned to a nebular phase dominated by forbidden transitions of iron-peak elements. ${ }^{35}$ If the identification of OI $\lambda 7774$ is correct and there is unburned oxygen at low velocity, [O I] $\lambda 6300$ should be a prominent feature in the nebular spectra of these objects. We searched for evidence of [O I] $\lambda 6300$ in spectra of SN 2005hk taken $\sim 400$ days after maximum, but SN 2005hk did not enter a nebular phase even at these late epochs and there was no evidence for $\left[\mathrm{O}_{\mathrm{I}}\right] \lambda 6300$.

Spectroscopy is no longer feasible after these late epochs because the SN is too faint. Instead, we use photometry to constrain the strength of the $[\mathrm{O} \mathrm{I}]$ line at +573 days after maximum. [O I] $\lambda 6300$ is near the center of the $r$ band (F625W) and is reasonably isolated from any other spectral features expected to be present. Therefore, we use the $r$-band photometric flux as a proxy for the flux in the oxygen line. If the $\left[\mathrm{O}_{\mathrm{I}}\right] \lambda 6300$ line began to dominate other spectral features and the nearby

\footnotetext{
35 Branch et al. (2008) argue that permitted lines dominate optical spectra of normal SNe Ia as late as a few months past maximum light, though by about 160 days past maximum typical SNe Ia have nebular spectra (Silverman et al. 2013).
}

continuum, as is predicted by the pure deflagration models (Kozma et al. 2005), we would expect a strong $r-i$ and $V-r$ color change as the SN transitions to a nebular phase.

To measure this color change, we examine two epochs of HST photometry of SN 2008A: the first epoch is at +396 days, at which we expect no contribution from [O I] $\lambda 6300$ based on our spectra of SN 2005hk at similar epochs. In our second observation at +573 days, we do see a strong $r-i$ color change, but $V-r$ remains relatively unchanged (see the discussion in Section 3.5); this cannot be easily explained by just the appearance of a strong line in the $r$ band.

Our photometry of SN 2008A at +573 allows us to quantitatively constrain the amount of oxygen in the ejecta below the critical density of $10^{6.5} \mathrm{~cm}^{-3}$. The complete deflagration models of Kozma et al. (2005) give us a relationship between the oxygen mass and the $\left[\mathrm{O}_{\mathrm{I}}\right] \lambda 6300$ line flux. If we unrealistically assume that all of the observed flux in $\mathrm{F} 625 \mathrm{~W}$ is from an oxygen line, we would derive a mass of $0.63 M_{\odot}$ of oxygen at low density. In reality, the oxygen line flux is only part of the observed broad-band F625W photometry. If we extrapolate just the $r$-band photometry from earlier times to day 573 , the data allow for only $0.40 M_{\odot}$ of oxygen to contribute additional line flux.

However, we can derive much more stringent oxygen mass limits if we constrain the SN spectral energy distribution (SED) in this wavelength region. Based on the observed spectroscopy at earlier times, we see that $\mathrm{SNe}$ Iax remain pseudo-continuum dominated in broad-band photometry to $\sim 400$ days past maximum, and the subsequent photometry does not show dramatic changes in late-time behavior among the different passbands. 


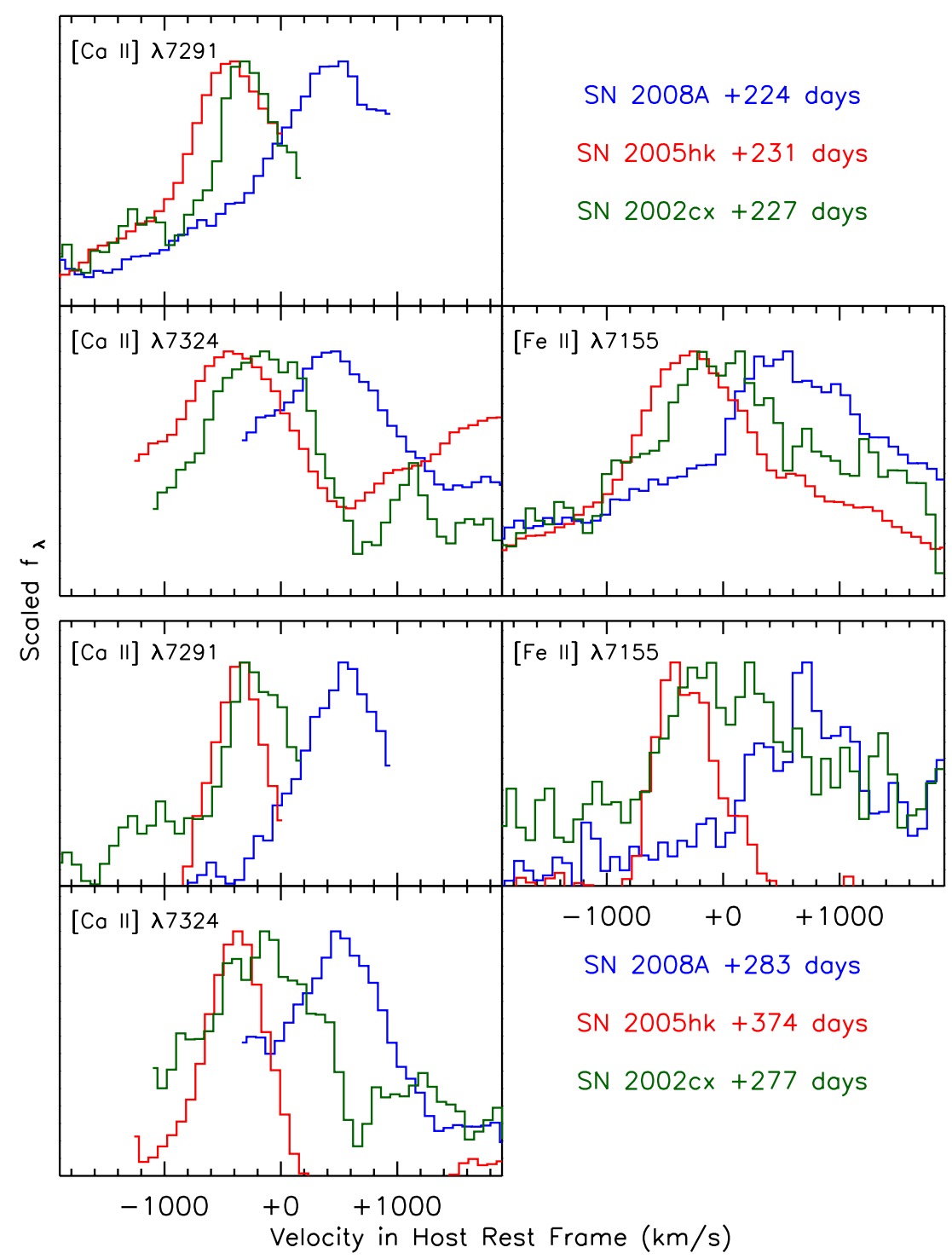

Figure 10. Velocity structure of the forbidden lines [Ca II] $\lambda 7291$, [Ca II] $\lambda 7323$, and [Fe II] $\lambda 7155$ in SN 2002cx (green; Jha et al. 2006a), SN 2005hk (red; Sahu et al. 2008 , and this work), and SN 2008A (blue; this work). The top three panels show each line at $\sim 230$ days past $B$ maximum and the bottom three panels show the same lines at $\sim 300$ days past $B$ maximum. The velocities of features are shown relative to the host-galaxy rest frame (in contrast to other figures, which are in the SN rest frame defined by these narrow lines). The host redshifts are $z=0.023963 \pm 0.000087$ for SN 2002cx in CGCG 044-035 (Meyer et al. 2004; Wong et al. 2006), $z=0.012993 \pm 0.000041$ for SN 2005hk in UGC 272 (Meyer et al. 2004; Wong et al. 2006), and $z=0.016428 \pm 0.000027$ for SN $2008 \mathrm{~A}$ in NGC 634 (Theureau et al. 1998). The line features of SN 2002cx and SN 2005hk are blueshifted compared to their hosts, while the features from SN 2008A are redshifted, with all the objects showing offsets of $\sim 400 \mathrm{~km} \mathrm{~s}^{-1}$. The features also show a significant decrease in velocity width over time.

(A color version of this figure is available in the online journal.)

This implies that we can use our measured F555W and F775W flux to bracket and constrain the expected continuum flux in F625W, with any new oxygen line flux showing up as an excess.

To do this we model the SN SED as a linear continuum (in logarithmic wavelength; $f_{\lambda} \propto \log \lambda$ ) plus a Gaussian line profile at $6300 \AA$ with a width of $500 \mathrm{~km} \mathrm{~s}^{-1}$, with a line flux calibrated to the oxygen mass as above (Kozma et al. 2005). We perform synthetic photometry on the resulting spectra to compare to our HST/ACS observations in F555W, F625W, and F775W. Essentially, the F555W and F775W data determine the continuum normalization and slope, and the F625W photometry then constrains the oxygen line flux. Our broad-band measurements are insensitive to the exact shape of the line profile, and using the three bands together, we marginalize over the nuisance parameters of the continuum slope and normalization to derive the oxygen mass limit.
Our constraint is shown in Figure 12; we find that the mass of oxygen below the critical density of [O I] $\lambda 6300$ is $<0.14 M_{\odot}$ at $95 \%$ confidence. Complete deflagration models that fully disrupt the white dwarf predict $\sim 0.4 M_{\odot}$ of unburned oxygen (Kozma et al. 2005). This is ruled out at high confidence $(>5 \sigma)$. Either a large amount of unburned material is not present in $\mathrm{SNe}$ Iax, or it remains at high density to very late epochs. Even if the ejecta mass were as low as $\sim 0.5 M_{\odot}$, we would expect $\sim 0.15 M_{\odot}$ of unburned oxygen (assuming the same ratio of oxygen mass to ejecta mass as the models from Kozma et al. 2005) — and this would still be ruled out at $>95 \%$ confidence.

As discussed, our results depend on the assumed shape of the SN SED. However, if we enforce a smooth continuum over the observed wavelength range (so we can incorporate the F555W and $\mathrm{F} 775 \mathrm{~W}$ data into our analysis), different prescriptions for 


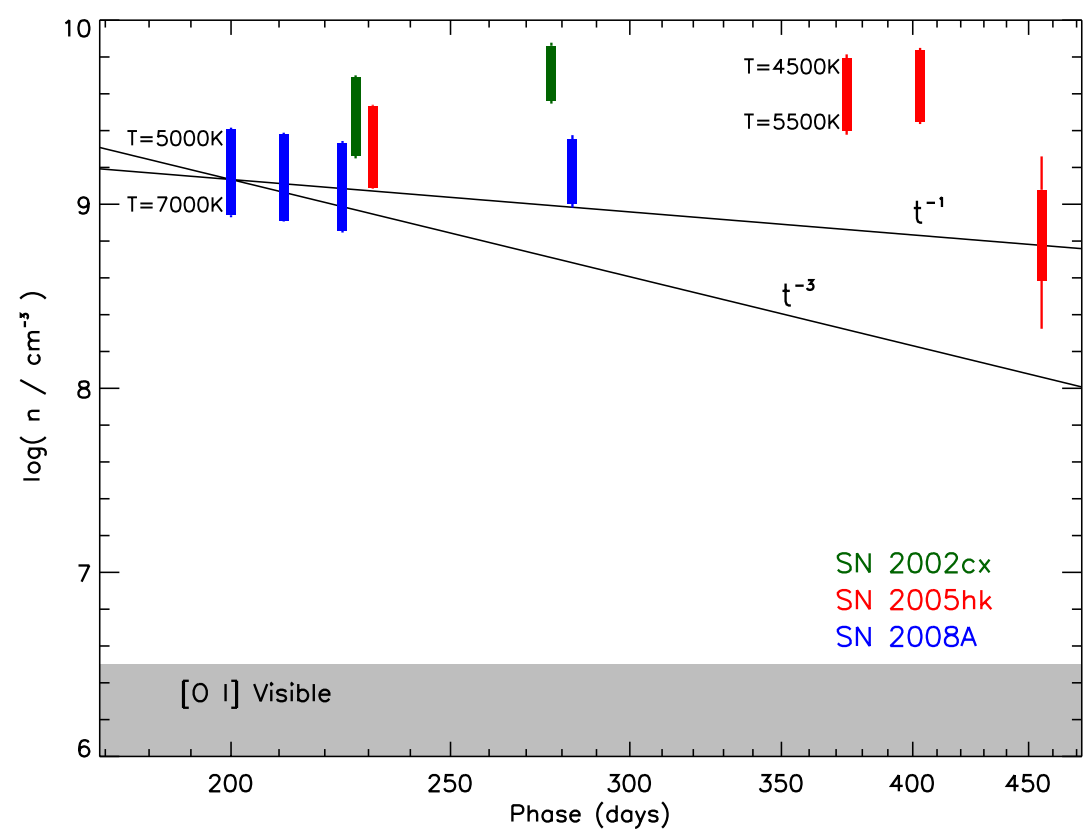

Figure 11. Density evolution of SN 2002cx (green), SN 2005hk (red), and SN 2008A (blue) using the measured forbidden to permitted line ratio of Ca II, based on the results of Ferland \& Persson (1989). The line ratio does not give a unique temperature and density, so the allowed temperature range was constrained by the presence of both Fe I and Fe II features, yielding the bands of allowed electron densities. Each object has a electron density of $\sim 10^{9} \mathrm{~cm}^{-3}$ at $\sim 230$ days past maximum light. The electron density is changing very slowly in all three objects; the results are consistent with no change in the electron density producing the observed emission. A density decrease proportional to $t^{-3}$ that would be predicted by simple homologous expansion models is not consistent with the measured density evolution of these three SNe.

(A color version of this figure is available in the online journal.)

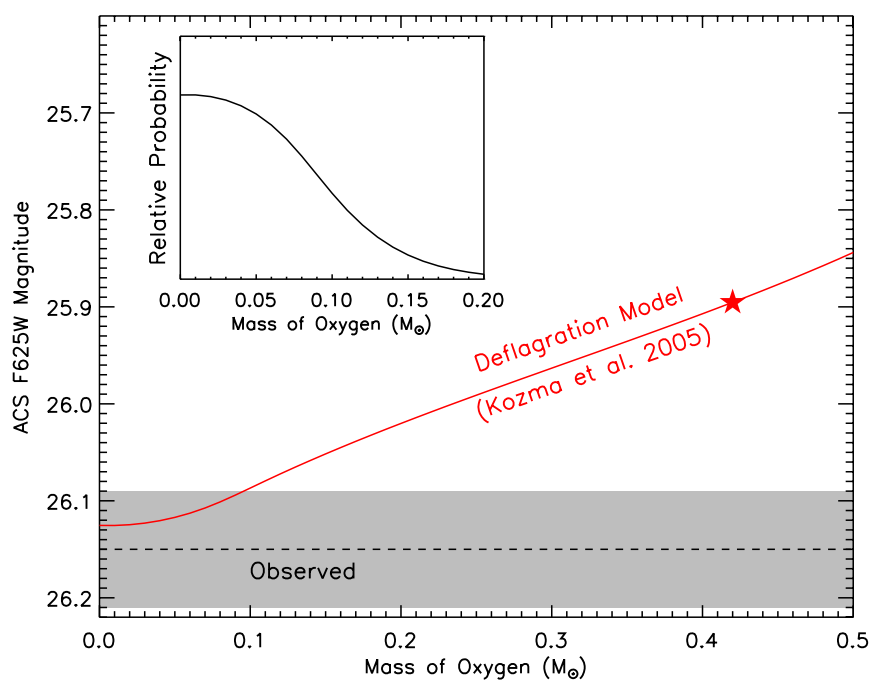

Figure 12. Constraints on low-density oxygen from HST/ACS photometry of SN 2008A at +573 days past maximum light. The gray region represents the $1 \sigma$ uncertainty in our measured photometry. The red line shows the predicted $r$-band magnitude (with flux from the [O I] $\lambda 6300$ line) assuming different amounts of unburned oxygen based on the models from Kozma et al. (2005). To calculate the probability density function (PDF) shown in the inset, we model the SED with a linear continuum and a Gaussian line profile centered at $6300 \AA$. We calculate the likelihood of the measured optical photometry (in F555W, $\mathrm{F} 625 \mathrm{~W}$, and F775W) and marginalize over the slope and normalization of the continuum to derive our final PDF. The PDF peaks at zero line flux, and puts a $95 \%$ confidence upper limit of $0.14 M_{\odot}$ of oxygen below the critical density for $[\mathrm{O}$ I $] \lambda 6300$. The pure deflagration model of Kozma et al. (2005) predicts $0.42 M_{\odot}$ of low-density unburned oxygen, which is ruled out at $>5 \sigma$.

(A color version of this figure is available in the online journal.)

the exact continuum shape lead to only small changes in the oxygen mass constraint of $\sim 0.02 M_{\odot}$.

Based on our temperature and density measurements, the electron density may have been significantly higher than the critical density for [O I] $\lambda 6300$ of $10^{6.5} \mathrm{~cm}^{-3}$ during our latest HST measurement of SN 2008A, nearly 600 days past maximum brightness. But if the roughly constant observed density is somehow the result of a receding photosphere, this would imply a significant amount of material at higher velocities and lower densities, and any oxygen mixed there should be detectable in [O I] emission as probed by our photometry. Complete deflagration models such as those of Kozma et al. (2005) predict strong mixing, so we disfavor the idea that all the oxygen is at low velocity, shielded by a photospheric "iron curtain" at higher velocity (Leonard 2007). Sahu et al. (2008) find in their models of SN 2005hk that if a significant fraction of the gamma-rays are still exciting photospheric transitions, the resulting [O I] $\lambda 6300$ is weak enough at +250 days to be consistent with the nondetection in the observations, even in the case of $0.8 M_{\odot}$ of unburned oxygen. However, it is unclear whether this can be sustained as late as +600 days, where our observations of SN 2008A otherwise significantly constrain any low-density oxygen. It is possible that this points to a diversity between objects like SN 2005hk and SN 2008A, but even for SN 2005hk itself, the suppression of [OI] emission must continue until at least +456 days past maximum, as it is undetected in our latest spectrum. Our conclusion is that either the spectroscopic detection of permitted O I $\lambda 7774$ at low velocity in SNe 2002cx and 2008A (as seen in Figure 9) is a misidentification, or, if not, a more complicated model (perhaps like one that produces a bound remnant) must be developed to explain the apparent contradiction between the permitted and forbidden lines; we discuss further implications of this finding in Section 4.

\subsection{IR Catastrophe}

The "infrared catastrophe" is a long-predicted, generically expected phenomenon that has nonetheless never been observed 
in normal SNe Ia (Leloudas et al. 2009). ${ }^{36}$ Axelrod (1980) calculated that once the ejecta temperature dropped below a critical value $\sim 1500 \mathrm{~K}$, there would be a radical redistribution of energy from the optical to the far-IR, dominated by finestructure lines of iron like [Fe I] $24 \mu \mathrm{m}$ and [Fe II] $26 \mu \mathrm{m}$ rather than the forbidden iron-peak lines seen in the optical and near-IR that dominate the SN Ia bolometric luminosity a few hundred days past maximum light (Sollerman et al. 2004). This redistribution of energy has been used to explain the line emission of SN 1987A (Kozma \& Fransson 1998), but it has never been observed in a normal SN Ia. One possible explanation is that the ejecta of SNe Ia stay above this critical temperature until after they are too faint to observe. Leloudas et al. (2009) find that even 785 days after $B$-band maximum brightness, SN 2003hv is not compatible with an IR catastrophe.

$\mathrm{SNe}$ Iax are unique testing grounds for this phenomenon. Because these objects remain at high density for so long, we might expect them to have enhanced cooling compared to normal SNe Ia. This could cause the IR catastrophe to happen early enough that it would still be feasible to image the SN. Qualitatively, if we use the excitation temperature of the iron (and assume microscopic mixing), we can estimate what the temperature of the ejecta will be at the time of our final HST measurement. Constructing a simple model that assumes that the excitation temperature of iron is $\sim 10,000 \mathrm{~K}$ at maximum brightness (giving rise to the dominant Fe III lines observed) and $\sim 4000 \mathrm{~K}$ at 300 days after maximum brightness (see Section 3.3), it is plausible (though by no means required) that the temperature at the epoch of our final HST measurement could approach the $\sim 1500 \mathrm{~K}$ threshold for the IR catastrophe. Sollerman et al. (2004) model this transition and find that the most easily observable signature (i.e., not in the far-IR) for the IR catastrophe is a dramatic color change in the near-IR, but we only have one near-IR measurement, (J:F110W), so we cannot observe this color change directly.

Sollerman et al. (2004) also show that the optical colors should become significantly redder in all optical bands during and after the IR catastrophe, with significant $V-R$ and $R-I$ color changes of $\sim 1$ mag. We compare this model with the observations of SN 2008A in Figure 13. Though there is some freedom to shift to model predictions vertically in magnitude and horizontally in time on the plot, we nonetheless see that the colors of SN 2008A change in the opposite way of the predicted color changes. Rather than getting redder, $V-I$ and $R-I$ data are bluer at late times (with $V-R$ remaining basically unchanged). Thus, we find no evidence for an IR catastrophe in SN 2008A, out to nearly 600 days past maximum light, despite the high densities.

The late-time spectra of SNe Iax differ significantly from those of normal SNe Ia. Normal SNe Ia cool through forbidden iron lines in the nebular phase. SNe Iax have not been shown to ever become nebular, and the strongest forbidden lines are from [Ca II]. There are iron features in the late-time spectra of SNe Iax, but a significant fraction of the emergent radiation is produced by permitted transitions. It is not even clear that radiative cooling dominates over adiabatic expansion at late times in SNe Iax. Because of these differences, it is possible that the predictions of IR catastrophe models for normal SNe Ia do not apply to SNe Iax. Ironically, it could be that the high densities that should enhance the cooling and lead to an earlier IR catastrophe

\footnotetext{
36 Taubenberger et al. (2011) suggest that the IR catastrophe could be at play in the rapid late-time decline of the light curve of the "super-Chandrasekhar" SN 2009dc.
}
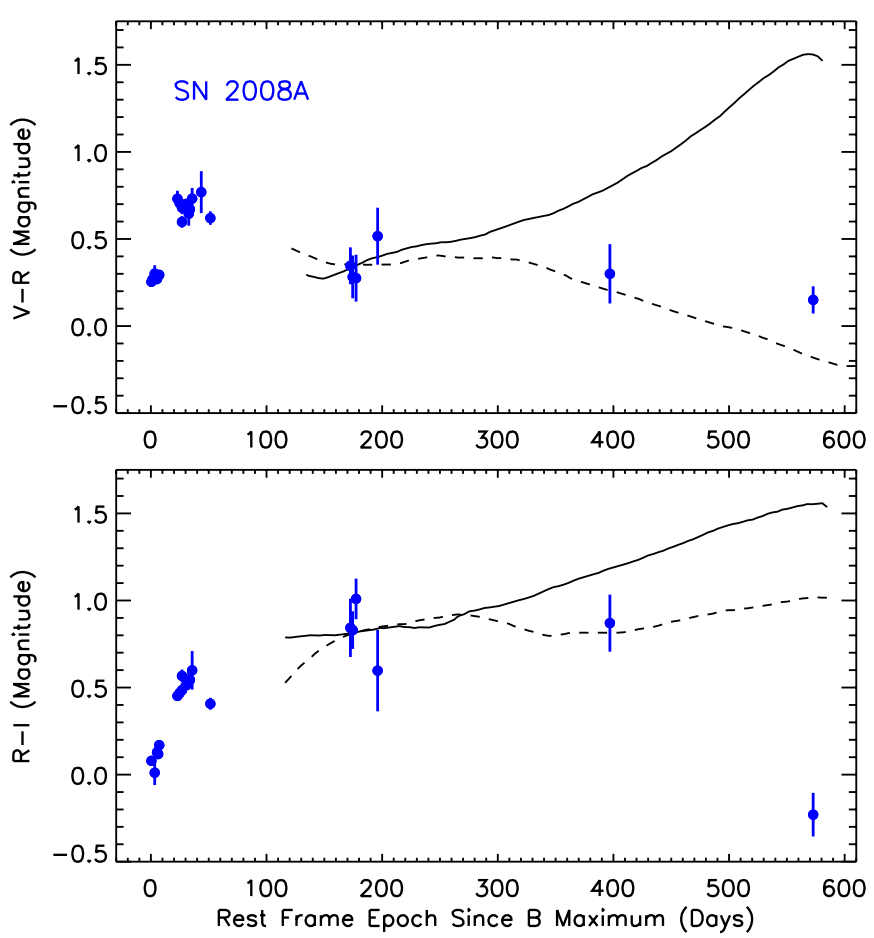

Figure 13. $V-R$ (top) and $R-I$ (bottom) color evolution of SN 2008A compared to IR-catastrophe models from Sollerman et al. (2004). The dashed line includes photodisintegration in the model while the solid line does not. The data have been standardized to $V, R$, and $I$ as in Figure 7. The models have been shifted vertically to match the data at $\sim 200$ days.

(A color version of this figure is available in the online journal.)

are instead responsible for quenching the expected changes in forbidden-line emission.

\subsection{Bolometric Luminosity}

We use ground-based and $H S T$ photometry to estimate the UV/optical/near-IR luminosity $L_{\text {UVOIR }}$ of SN 2002cx, SN 2005hk, SN 2008A, and the normal SN Ia 2003hv. Sollerman et al. (2004) and Leloudas et al. (2009) show that the flux in the near-IR becomes a significant fraction of the bolometric luminosity of SNe Ia at late times, and we are able to replicate the near-IR corrections of Leloudas et al. (2009) in our calculations for SN 2003hv.

For SNe 2002cx and 2008A, we do not have much information about the flux in the IR. Phillips et al. (2007) present near-IR light curves of SN 2005hk to a few months past maximum light, showing significantly enhanced near-IR flux compared to normal SNe Ia. However, at these epochs the near-IR contribution to the bolometric luminosity is only a few percent, and it is unclear whether the excess near-IR flux persists to later epochs, or merely becomes more significant earlier in SNe Iax than in normal SNe Ia.

To correct the SNe Iax for the poorly constrained near-IR flux, we integrate our interpolated photometric SED from $3000 \AA$ to $10,000 \AA$ and add the IR contribution fraction measured for SN 2003hv (Leloudas et al. 2009). This approach produces results that are consistent with the late-time near-IR data we do have (a WFC3/IR F110W measurement for SN 2008A and NICMOS F110W and F160W upper limits for SN 2005hk; Table 4). For the final two HST observations of SN 2005hk, we have a detection only in a single optical photometric band; to calculate the bolometric luminosity at these late epochs, we use 


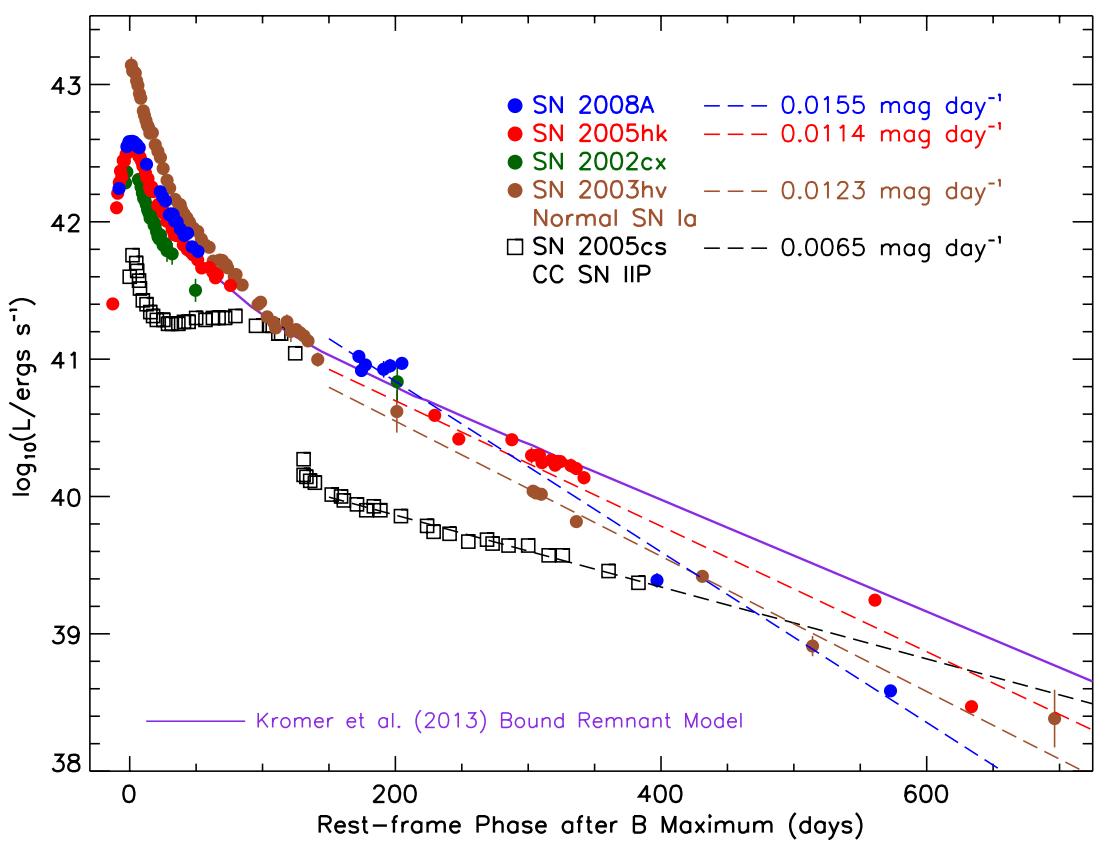

Figure 14. Bolometric light curves of SN 2002cx (Li et al. 2003; Jha et al. 2006a), SN 2005hk (Phillips et al. 2007; Sahu et al. 2008, and data from SDSS-II), SN 2008A (Ganeshalingam et al. 2010; Hicken et al. 2012, and this work), the normal Type Ia SN 2003hv (Leloudas et al. 2009), and the Type IIP SN 2005cs (Pastorello et al. 2009). The bolometric luminosities of SNe Iax are similar to those of normal SNe Ia starting a few months after maximum, but differ significantly from those of SNe IIP. SN 2003hv declines much faster than SN 2008A and its counterparts at $\sim 200$ days past maximum. The magenta line shows the model from Kromer et al. (2013) that assumes complete gamma-ray trapping. This model fits most of the data well, but it is not consistent with the latest measurements of SN 2008A and the last measurement of SN 2005hk.

(A color version of this figure is available in the online journal.)

the SED of SN 2008A at a similar phase and scale the flux to match the SN 2005hk observation.

As shown in Figure 14, we find that the bolometric light curves of SNe Iax are markedly different from that of a typical core-collapse SN IIP, SN 2005cs (Pastorello et al. 2009), though of course core-collapse SNe show a wide range of behavior. The SNe Iax are similar to each other, and significantly less luminous than the normal SN Ia $2003 \mathrm{hv}$ at maximum light, but broadly similar to SN 2003hv starting $\sim 100$ days after maximum light.

The faster decline rate of SN 2003hv compared to SNe Iax for the first 200 days suggests that the escape fraction of gammarays in normal SNe Ia increases more quickly than in SNe Iax. This may result from a more rapid decrease in the density of the ejecta in normal SNe Ia (also suggested by our inferred SNe Iax densities; Figure 11). The bolometric luminosity of SN 2005hk roughly follows the full gamma-ray trapping model of Kromer et al. (2013) for all but the latest epoch. SN 2008A is also consistent with the full gamma-ray trapping model at early times.

However, in the latest observations, more than $\sim 400$ days past maximum, that trend reverses: SN 2003hv declines more slowly than SN 2005hk or SN 2008A. At these epochs, the decline rate for SN $2003 \mathrm{hv}$ is close to the predicted ${ }^{56} \mathrm{Co}$ to ${ }^{56} \mathrm{Fe}$ decay rate of $0.0098 \mathrm{mag} \mathrm{day}^{-1}$. This may be an indication of positron trapping in the ejecta of this normal SN Ia. About 3\% of the ${ }^{56} \mathrm{Co}$ decay energy goes to positrons, compared to $97 \%$ in gamma-rays (Milne et al. 1999). Even if the gamma-ray escape fraction is near unity, positrons may be trapped by (nonradial) magnetic fields, and can lead to a shallowing of the bolometric luminosity decline rate when the luminosity falls to $\sim 3 \%$ of the full gamma-ray trapping prediction.

At the epochs of our last observations of both SNe 2005hk and 2008A, the bolometric luminosity drops below that of the Kromer et al. (2013) model. This could be a sign that the gamma- ray escape fraction is beginning to increase, at a much later epoch than occurs for normal SNe Ia because of the much higher densities in the ejecta. We might then predict that at even later times, again when the bolometric luminosity falls to $\sim 3 \%$ of the full gamma-ray trapping prediction, the bolometric light curves of SNe Iax may flatten because of positron trapping. Nonetheless, this remains speculative, as we are not measuring the true bolometric luminosity. If either normal $\mathrm{SNe}$ Ia or SNe Iax undergo an IR catastrophe, the UVOIR luminosity will not trace the bolometric luminosity, complicating efforts to constrain the positron trapping. Moreover, many normal SNe Ia show steeper decline rates at late epochs, inconsistent with full positron trapping (e.g., Lair et al. 2006), and in some cases even when the near-IR flux is included in the "bolometric" luminosity (Stanishev et al. 2007a).

\section{DISCUSSION}

As described in Section 3.2, the velocity shifts measured from late-time forbidden lines in SNe 2002cx, 2005hk, and 2008A are larger than can be accounted for by galactic rotation alone. These offsets are measured from [Ca II] and [Fe II] lines; if the ejecta were optically thin in these lines as expected, the velocity offsets could be caused by bulk velocity shifts of the ejecta. This might correspond to the high-velocity remnant predicted by the failed deflagration model of Jordan et al. (2012), although significant velocity kicks are not found in similar models from Kromer et al. (2013) or Fink et al. (2014).

The velocity shifts could also be the result of an asymmetric explosion. However, Chornock et al. (2006) measured less than $1 \%$ polarization for SN $2005 \mathrm{hk}$, implying very little asymmetry at early times (see also Maund et al. 2010). For the normal Type Ia SN 2003hv, Leloudas et al. (2009) measured a velocity shift of $\sim 2600 \mathrm{~km} \mathrm{~s}^{-1}$ in the [Fe II] $\lambda 8617$ line. Maeda et al. 
(2010b) show that the large velocity shifts in the inner regions of SN 2003hv could arise in the deflagration phase of a delayed detonation model. Maeda et al. (2010a) use a model with asymmetry of the deflagration phase to explain the variation of SN Ia velocity gradients, and show that different viewing angles can account for much of the diversity of SNe Ia (but see Wang et al. 2013, who find a host-galaxy dependence that cannot be explained by viewing angle alone). In this model, the deflagration stage is characterized by turbulent burning underlying a convective, bipolar structure which creates an asymmetry in the ejecta (Kuhlen et al. 2006; Röpke et al. 2007). The velocity shifts for SNe 2005hk and SN 2008A are much smaller than that for SN 2003hv, which might be caused by a lower kinetic energy during the deflagration phase, and perhaps implying less total burning. The fact that the forbidden-line velocity offsets are comparable to line widths in both SNe Iax and normal $\mathrm{SNe}$ Ia may point to a common physical origin, though this may be generic to all $\mathrm{SNe}$.

The identification of S II and Si II lines in early-time spectra of SNe Iax point to a thermonuclear event (Foley et al. 2010a, 2013). Our identification of late-time [Ni II] $\lambda 7378$ in SN 2005hk and SN 2008A could thus have important consequences, as Maeda et al. (2010b, 2010a) identify both [Fe II] $\lambda 7155$ and [Ni II] $\lambda 7378$ as lines that correspond to deflagration ashes. They argue that these lines trace material subject to long exposure to low heat, as opposed to [Fe III] $\lambda 4701$ that traces the detonation phase. This [Fe III] transition is not obvious in SN Iax spectra at late times, which may imply that there is no transition to a detonation.

What could suppress a transition to a detonation in SNe Iax? From simulations, Röpke et al. (2007) suggest that the number and position of deflagration ignition points could be a significant factor. For example, they claim that a model with two overlapping ignition bubbles at the center of the white dwarf was unlikely to produce a detonation; thus, such a scenario might produce a SN Iax. However, Seitenzahl et al. (2013) find that the conditions are met for a detonation in all of their models ranging from a single ignition point up to 1600 ignition points. Even a pure deflagration does not explain the incredibly low kinetic energy for the most extreme members of the SN Iax class, such as SN 2008ha.

To explore low-energy complete deflagration scenarios for more typical SNe Iax, we examined a homologous expansion model using the density profiles of Röpke (2005). We find that it is possible to produce the high densities observed at late times, but the initial kinetic energy distribution must be several times lower than in the model (also assuming the emitting region moves to lower velocities and roughly constant density, as Figure 11 requires). Scaling down the velocity of the density distribution, we find that the speed of material at the radius with $10 \%$ of the total enclosed mass $\left(R_{0.1}\right)$ must be as low as $150 \mathrm{~km} \mathrm{~s}^{-1}$ to approach the observations. At first glance, this result seems like it could qualitatively explain the lack of the [OI] $\lambda 6300$ flux by keeping a substantial amount of unburned material at high density. However, quantitatively, we find that even for homologous expansion that is scaled to the lower kinetic energies, at 600 days after maximum brightness, $\sim 55 \%$ of the oxygen should still be below the critical density. This corresponds to $\sim 0.2 M_{\odot}$ of low-density oxygen in the Röpke (2005) models, and is ruled out by our observations at high significance (Figure 12). However, our constraint could be weakened if the [OI] excitation is being suppressed by a mechanism where photospheric lines are preferentially excited
(Sahu et al. 2008; see Section 3.4), though it is unclear whether this could continue as late as 600 days past maximum light.

Generically, we find that even if there is a photosphere hiding the innermost regions, the models predict oxygen at higher velocities that should be visible, and yet is not seen. Observationally, the decreasing widths of the forbidden lines of iron and calcium with time (see Figure 10) imply that these lines are not being excited everywhere in the ejecta. If these lines are only produced near the "photosphere" responsible for the numerous permitted $\mathrm{Fe}$ lines, there must be a large reservoir of lower-density material at higher velocities that is not radiating either in these lines, nor is it seen in lower density tracers like [O I] $\lambda 6300$.

In normal SNe Ia, the widths of nebular lines at late times is of order $10^{4} \mathrm{~km} \mathrm{~s}^{-1}$ (Mazzali et al. 1998; Silverman et al. 2013). If, as expected, we are "seeing through" all the excited ejecta in these forbidden transitions, the line profiles directly reveal the velocity structure, and the observed line width is effectively the "final" velocity for the ejecta, $v_{\infty}$. Moreover, for an exploding white dwarf that is completely disrupted, we expect $v_{\infty} \gtrsim v_{\text {esc }}$, the escape velocity of that material in the white dwarf; otherwise the explosion would need to be finely tuned to barely unbind the white dwarf and leave the ejecta expanding at low velocities.

The widths of forbidden lines of $\mathrm{Ca}$ II and Fe II in the late-time spectra of SN $2005 \mathrm{hk}$ are unprecedentedly low- $500 \mathrm{~km} \mathrm{~s}^{-1}$. If this is interpreted as the velocity at infinity for the emitting ejecta, it is an order of magnitude below the escape velocity from the surface of the white dwarf, $v_{\infty} \ll v_{\text {esc }}$. Using a simple model of a Chandrasekhar-mass white dwarf with a polytropic equation of state, we calculated the escape velocity as a function of the enclosed mass throughout the white dwarf. At $v_{\text {esc }}=500 \mathrm{~km} \mathrm{~s}^{-1}$ the enclosed mass is just $\sim 5 \times 10^{-6}$ of the total mass. It seems implausible that all of the late-time emission arises from just this innermost tiny fraction of the white dwarf.

More likely, we are witnessing a weak explosion, in which the explosion energy is close to or less than the binding energy of the white dwarf. The material emitting in the forbidden lines was barely unbound, and we observe it at low $v_{\infty} \ll v_{\text {esc }}$. In that case, unless again the explosion energy is finely tuned, we would also expect a significant fraction of the original white dwarf to not reach escape velocity, and remain bound, leaving behind a remnant. The high densities observed might also be explained if the white dwarf was not fully disrupted, with the mixed composition of the unbound ejecta arising from a deflagration. Livne et al. (2005) show that a single ignition that is offset from the center of the white dwarf could form a bubble that would convectively rise and break through the surface, without fusion outside of the bubble. Events like this could explain typical SNe Iax and the extreme SN 2008ha, yet still show the thermonuclear signature of Si II and S II.

Kromer et al. (2013) and Jordan et al. (2012) find that a failed deflagration of a white dwarf can produce properties similar to those observed for SNe 2005hk and 2008A. A key prediction of these models is that the explosion leaves behind a bound remnant. This is consistent with our argument above about the escape velocity of the white dwarf. Jordan et al. (2012) find that the remnant receives a kick of $\sim 500 \mathrm{~km} \mathrm{~s}^{-1}$, which is roughly consistent with our measurements of the velocity offsets from the host galaxies. This assumes that the velocities of the forbidden lines are measuring the bulk motion of the ejecta rather than an excitation effect. While Kromer et al. (2013) do not find such significant kick velocities, their model matches the 


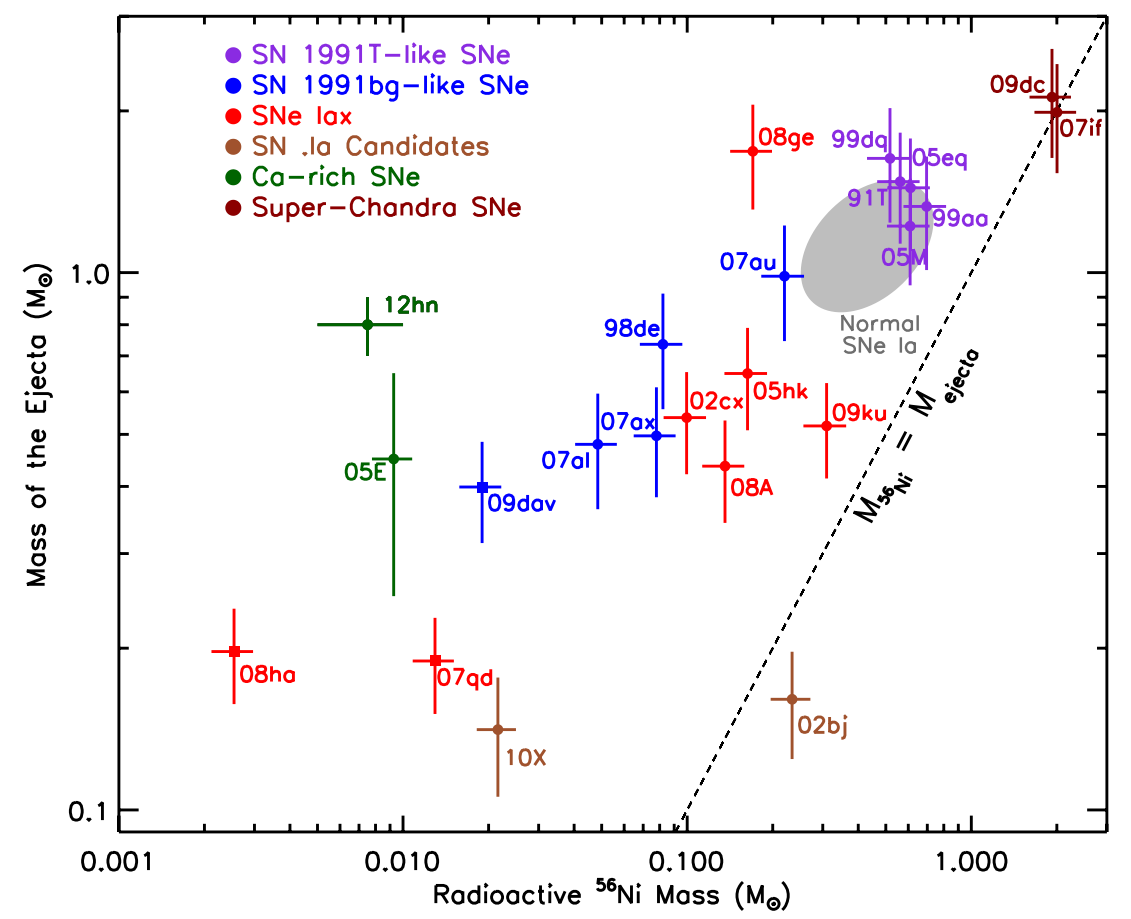

Figure 15. Total ejected mass vs. radioactive nickel mass of white-dwarf SNe. Subclasses of objects are grouped by color as indicated in the plot. Circles denote typical members of the subclass, while squares show more extreme members. The dashed line denotes where the ejected mass equals the radioactive nickel mass. Objects to the right of the line (like SN 2002bj) require additional energy sources (beyond ${ }^{56} \mathrm{Ni}$ radioactive power) to explain their luminosity. References: SN 1991T (Filippenko et al. 1992b; Phillips et al. 1992); SN 1998de, SN 2007al, SN 2007au, and PTF 2009dav (Sullivan et al. 2011); SN 1999aa and SN 1999dq (Jha et al. 2006b); SN 2002bj (Poznanski et al. 2010); SN 2002cx (Li et al. 2003; Jha et al. 2006a); SN 2003du (Hicken et al. 2009); SN 2003hv (Leloudas et al. 2009); SN 2005E (Perets et al. 2010; Waldman et al. 2011); SN 2012hn (Valenti et al. 2014); SN 2005M and SN 2005eq (Ganeshalingam et al. 2010); SN 2005hk (Phillips et al. 2007); SN 2007ax (Kasliwal et al. 2008); SN 2007if (Scalzo et al. 2010); SN 2007qd (McClelland et al. 2010); SN 2008A (this work); SN 2008ge (Foley et al. 2010b); SN 2008ha (Foley et al. 2009; Valenti et al. 2009); SN 2009dc (Taubenberger et al. 2011); SN 2009ku (Narayan et al. 2011); and SN 2010X (Kasliwal et al. 2010). Ancillary data were used in the analysis, including ejecta velocities (Foley et al. 2011), rise times (Ganeshalingam et al. 2011), bolometric corrections (Contardo et al. 2000), and distances and luminosities (Ganeshalingam et al. 2013).

(A color version of this figure is available in the online journal.)

bolometric light curves of SN 2005hk and SN 2008A relatively well, except at the latest observed epochs (Figure 14).

While suggestive, the case for a bound remnant is not without problems. If we assume homologous expansion, material at $300 \mathrm{~km} \mathrm{~s}^{-1}$, about half the width of the forbidden lines, would be at $100 \mathrm{AU}$ about 600 days past explosion. This cannot be the radius of a true photosphere, because the bolometric luminosity at that epoch $\left(\sim 10^{38} \mathrm{erg} \mathrm{s}^{-1}\right)$ is much too low given the estimated temperature. The bolometric luminosity limits the photospheric radius to $\lesssim 3.5 \mathrm{AU}$, making it difficult to directly connect the high density expanding material with a potential bound remnant. Moreover, these models still require an explanation for the lack of unburned material detected in ejecta that should be well mixed (including intermediate-mass and iron-group elements) and reach low density. Perhaps additional complexity is required, such as back-warming in which inwardly traveling gammarays deposit their energy and heat lower-velocity regions, while outwardly traveling gamma-rays escape.

Throughout this paper, we have assumed that radioactive heating from ${ }^{56} \mathrm{Ni}$ dominates the luminosity of these SNe Iax. It is instructive to put SNe Iax in context with other probable thermonuclear explosions of white dwarfs. We compiled a sample of such objects from the literature to infer their radioactive nickel mass and ejecta mass; the results are shown in Figure 15. The ${ }^{56} \mathrm{Ni}$ mass was calculated using the Arnett (1982) rule, and for this comparison we simply adopted a uniform uncertainty of $15 \%$ for all bolometric luminosities and a $10 \%$ uncertainty for the SN rise times. These correspond to typical published un- certainties for the objects for which error bars were reported. We calculated the ejecta mass following Foley et al. (2009), assuming an opacity of $\kappa=0.1 \mathrm{~cm}^{2} \mathrm{~g}^{-1}$ and a uniform $5 \%$ uncertainty on the ejecta velocities. The relations are scaled such that normal SNe Ia produce $1.4 M_{\odot}$ of ejecta, with $\sim 0.5 M_{\odot}$ of ${ }^{56} \mathrm{Ni}$. Normal SNe Ia form an approximate continuum from the subluminous SN 1991bg-like objects (Filippenko et al. 1992a; Leibundgut et al. 1993) to the superluminous SN 1991T-like (Filippenko et al. 1992b; Phillips et al. 1992), and even to more extreme "super-Chandra" objects such as SN 2009dc (Howell et al. 2006; Scalzo et al. 2010; Taubenberger et al. 2011; Silverman et al. 2011).

The three SNe Iax studied in detail in this work cluster below the SN $1991 \mathrm{bg}$-like objects with $\sim 0.5 M_{\odot}$ of ejecta and $0.15 M_{\odot}$ of radioactive nickel. However, the $\mathrm{SNe}$ Iax class shows significant diversity in both axes: SN 2009ku is very close to having burned a large fraction of its ejecta into ${ }^{56} \mathrm{Ni}$, while SN 2008ge shows lower amounts of radioactive nickel than normal SNe Ia, but a similar ejecta mass. Two of the most extreme members of the SNe Iax subclass are SN 2008ha and SN 2007qd (McClelland et al. 2010); both events produced low ejecta mass and ${ }^{56} \mathrm{Ni}$ mass, similar to other low-luminosity transients like the "calcium-rich" SN 2005E (Perets et al. 2010) or the "SN.Ia" candidate SN 2010X (Bildsten et al. 2007; Kasliwal et al. 2010). Taken together, the luminosities, rise times, and ejecta velocities of SNe Iax certainly do not contradict the hypothesis that SNe Iax are thermonuclear explosions of white dwarfs in which radioactive nickel provides the UVOIR 
light, though the observations do pose a significant challenge to models. We note that Sahu et al. (2008) were able to fit the bolometric light curve and some of the spectral features of SN 2005hk with an explosion model that assumed an ejecta mass of $1.4 M_{\odot}$ which implies that more complex modeling may be necessary.

\section{SUMMARY}

We present ground-based and $H S T$ photometry and spectroscopy of SNe 2005hk and 2008A, two typical SNe Iax. These objects remain at high density for $\sim 2$ yr after explosion and are not observed to enter the typical nebular phase up to 400 days after $B$-band maximum brightness. We find no evidence for unburned material at low velocities, either directly through spectroscopy or indirectly with our HST photometry. Based on emission-line diagnostics, we find that the density of the emitting region remains roughly constant over the duration of the observations, though the widths of even the forbidden lines decrease. We do not see the signature of the IR catastrophe in optical colors. The bolometric luminosity of SN 2005hk and SN 2008A fades more slowly than that of normal SNe Ia at 100-200 days after maximum brightness, but then declines faster than in normal SNe Ia at phases of 400-600 days. Failed deflagration models that leave a bound remnant (e.g., Jordan et al. 2012; Kromer et al. 2013) show promise for explaining these explosions, but no single proposed model can explain all of our observations.

We dedicate this paper to the lasting memory of our dear friend and cherished colleague, Weidong Li.

We thank Mark Phillips and Roger Romani for help in acquiring the HST observations of SN 2005hk. We acknowledge usage of the NASA Extragalactic Database (http://ned.ipac.caltech.edu) and the HyperLeda database (http://leda.univ-lyon1.fr).

This research at Rutgers University was supported through NASA/HST grants GO-11133.01 and GO-11590.01, along with U.S. Department of Energy (DOE) grant DE-FG02-08ER41562, and National Science Foundation (NSF) CAREER award AST0847157 to S.W.J., and a GAANN Fellowship to C.M. J.M.S. is supported by an NSF Astronomy and Astrophysics Postdoctoral Fellowship under award AST-1302771. The research of J.C.W. is supported in part by NSF Grant AST-1109801. G.L. is supported by the Swedish Research Council through grant No. 623-2011-7117. A.V.F. and his group at UC Berkeley are funded by Gary and Cynthia Bengier, the Richard and Rhoda Goldman Fund, NSF grant AST-1211916, the TABASGO Foundation, and NASA/HST grants GO-10877 and AR-12623.

Support for HST programs GO-10877, GO-11133, GO11590, and AR-12623 was provided by NASA through a grant from the Space Telescope Science Institute, which is operated by the Association of Universities for Research in Astronomy, Incorporated, under NASA contract NAS5-26555.

Some of the data presented herein were obtained at the W. M. Keck Observatory, which is operated as a scientific partnership among the University of California, the California Institute of Technology, and NASA, made possible by the generous financial support of the W. M. Keck Foundation. The authors recognize and acknowledge the very significant cultural role and reverence that the summit of Mauna Kea has always had within the indigenous Hawaiian community, and we are most privileged to have the opportunity to explore the universe from this mountain.
Funding for the SDSS and SDSS-II has been provided by the Alfred P. Sloan Foundation, the Participating Institutions, the NSF, the U.S. Department of Energy, NASA, the Japanese Monbukagakusho, the Max Planck Society, and the Higher Education Funding Council for England. The SDSS Web site is http://www.sdss.org/. The SDSS is managed by the Astrophysical Research Consortium for the Participating Institutions. The Participating Institutions are the American Museum of Natural History, Astrophysical Institute Potsdam, University of Basel, University of Cambridge, Case Western Reserve University, University of Chicago, Drexel University, Fermilab, the Institute for Advanced Study, the Japan Participation Group, Johns Hopkins University, the Joint Institute for Nuclear Astrophysics, the Kavli Institute for Particle Astrophysics and Cosmology, the Korean Scientist Group, the Chinese Academy of Sciences (LAMOST), Los Alamos National Laboratory, the Max-PlanckInstitute for Astronomy (MPIA), the Max-Planck-Institute for Astrophysics (MPA), New Mexico State University, Ohio State University, University of Pittsburgh, University of Portsmouth, Princeton University, the United States Naval Observatory, and the University of Washington.

\section{REFERENCES}

Arnett, W. D. 1982, ApJ, 253, 785

Axelrod, T. S. 1980, PhD thesis, AA(California Univ., Santa Cruz.)

Bertin, E. 2012, in ASP Conf. Ser. 461, Astronomical Data Analysis Software and Systems XXI, ed. P. Ballester, D. Egret, \& N. P. F. Lorente (San Francisco, CA: ASP), 263

Bildsten, L., Shen, K. J., Weinberg, N. N., \& Nelemans, G. 2007, ApJL, 662, L95

Branch, D., Baron, E., Hall, N., Melakayil, M., \& Parrent, J. 2005, PASP, 117,545

Branch, D., Baron, E., Thomas, R. C., et al. 2004, PASP, 116, 903

Branch, D., Jeffery, D. J., Parrent, J., et al. 2008, PASP, 120, 135

Chiaberge, M., Lim, P. L., Kozhurina-Platais, V., Sirianni, M., \& Mack, J. 2009, Updated CTE Photometric Correction for WFC and HRC, Tech. Rep. (STSci)

Chornock, R., Filippenko, A. V., Branch, D., et al. 2006, PASP, 118, 722

Conley, A., Guy, J., Sullivan, M., et al. 2011, ApJS, 192, 1

Contardo, G., Leibundgut, B., \& Vacca, W. D. 2000, A\&A, 359, 876

Dolphin, A. E. 2009, PASP, 121, 655

Faber, S. M., Phillips, A. C., Kibrick, R. I., et al. 2003, Proc. SPIE, 4841, 1657 Ferland, G. J., \& Persson, S. E. 1989, ApJ, 347, 656

Filippenko, A. V. 2003, in From Twilight to Highlight: The Physics of Supernovae, ed. W. Hillebrandt \& B. Leibundgut (Berlin: Springer), 171

Filippenko, A. V., Li, W. D., Treffers, R. R., \& Modjaz, M. 2001, in ASP Conf. Ser. 246, IAU Colloq. 183: Small Telescope Astronomy on Global Scales, ed. B. Paczynski, W.-P. Chen, \& C. Lemme (San Francisco, CA: ASP), 121 Filippenko, A. V., Richmond, M. W., Branch, D., et al. 1992a, AJ, 104, 1543

Filippenko, A. V., Richmond, M. W., Matheson, T., et al. 1992b, ApJL, 384, L15

Fink, M., Kromer, M., Seitenzahl, I. R., et al. 2014, MNRAS, 438, 1762

Folatelli, G., Phillips, M. M., Morrell, N., et al. 2012, ApJ, 745, 74

Foley, R. J., Brown, P. J., Rest, A., et al. 2010a, ApJL, 708, L61

Foley, R. J., Challis, P. J., Chornock, R., et al. 2013, ApJ, 767, 57

Foley, R. J., Chornock, R., Filippenko, A. V., et al. 2009, AJ, 138, 376

Foley, R. J., Rest, A., Stritzinger, M., et al. 2010b, AJ, 140, 1321

Foley, R. J., Sanders, N. E., \& Kirshner, R. P. 2011, ApJ, 742, 89

Frieman, J. A., Bassett, B., Becker, A., et al. 2008, AJ, 135, 338

Fruchter, A., Sosey, M., Hack, W., et al. 2009, HST MultiDrizzle Handbook (Baltimore, MD: STScI)

Fukugita, M., Ichikawa, T., Gunn, J. E., et al. 1996, AJ, 111, 1748

Gamezo, V. N., Khokhlov, A. M., \& Oran, E. S. 2004, PhRvL, 92, 211102

Gamezo, V. N., Khokhlov, A. M., \& Oran, E. S. 2005, ApJ, 623, 337

Gamezo, V. N., Khokhlov, A. M., Oran, E. S., Chtchelkanova, A. Y., \& Rosenberg, R. O. 2003, Sci, 299, 77

Ganeshalingam, M., Li, W., \& Filippenko, A. V. 2011, MNRAS, 416, 2607

Ganeshalingam, M., Li, W., \& Filippenko, A. V. 2013, MNRAS, 433, 2240

Ganeshalingam, M., Li, W., Filippenko, A. V., et al. 2010, ApJS, 190, 418

Hatano, K., Branch, D., Fisher, A., Millard, J., \& Baron, E. 1999, ApJS, 121, 233 
Hicken, M., Challis, P., Jha, S., et al. 2009, ApJ, 700, 331

Hicken, M., Challis, P., Kirshner, R. P., et al. 2012, ApJS, 200, 12

Holtzman, J. A., Hester, J. J., Casertano, S., et al. 1995, PASP, 107, 156

Holtzman, J. A., Marriner, J., Kessler, R., et al. 2008, AJ, 136, 2306

Hook, R., \& Stoehr, F. 2008, WFC3 Support in Tiny Tim, Tech. Rep. (Baltimore, MD: STScI)

Howell, D. A., Sullivan, M., Nugent, P. E., et al. 2006, Natur, 443, 308

Jha, S., Branch, D., Chornock, R., et al. 2006a, AJ, 132, 189

Jha, S., Garnavich, P. M., Kirshner, R. P., et al. 1999, ApJS, 125, 73

Jha, S., Kirshner, R. P., Challis, P., et al. 2006b, AJ, 131, 527

Jordan, G. C., IV, Perets, H. B., Fisher, R. T., \& van Rossum, D. R. 2012, ApJL, 761, L23

Kasen, D. 2006, ApJ, 649, 939

Kasliwal, M. M., Kulkarni, S. R., Gal-Yam, A., et al. 2010, ApJL, 723, L98

Kasliwal, M. M., Ofek, E. O., Gal-Yam, A., et al. 2008, ApJL, 683, L29

Kessler, R., Becker, A. C., Cinabro, D., et al. 2009, ApJS, 185, 32

Khokhlov, A. M. 1991, A\&A, 245, 114

Kirshner, R. P., Jeffery, D. J., Leibundgut, B., et al. 1993, ApJ, 415, 589

Kozma, C., \& Fransson, C. 1998, ApJ, 497, 431

Kozma, C., Fransson, C., Hillebrandt, W., et al. 2005, A\&A, 437, 983

Krist, J. 1993, in ASP Conf. Ser. 52, Astronomical Data Analysis Software and Systems II, ed. R. J. Hanisch, R. J. V. Brissenden, \& J. Barnes (San Francisco, CA: ASP), 536

Kromer, M., Fink, M., Stanishev, V., et al. 2013, MNRAS, 429, 2287

Kuhlen, M., Woosley, S. E., \& Glatzmaier, G. A. 2006, ApJ, 640, 407

Lair, J. C., Leising, M. D., Milne, P. A., \& Williams, G. G. 2006, AJ, 132, 2024

Leibundgut, B., Kirshner, R. P., Phillips, M. M., et al. 1993, AJ, 105, 301

Leloudas, G., Stritzinger, M. D., Sollerman, J., et al. 2009, A\&A, 505, 265

Leonard, D. C. 2007, ApJ, 670, 1275

Li, W., Filippenko, A. V., Chornock, R., et al. 2003, PASP, 115, 453

Li, W., Filippenko, A. V., Treffers, R. R., et al. 2001, ApJ, 546, 734

Livne, E., Asida, S. M., \& Höflich, P. 2005, ApJ, 632, 443

Lyman, J. D., James, P. A., Perets, H. B., et al. 2013, MNRAS, 434, 527

Maeda, K., Benetti, S., Stritzinger, M., et al. 2010a, Natur, 466, 82

Maeda, K., Taubenberger, S., Sollerman, J., et al. 2010b, ApJ, 708, 1703

Maguire, K., Jerkstrand, A., Smartt, S. J., et al. 2012, MNRAS, 420, 3451

Maund, J. R., Wheeler, J. C., Wang, L., et al. 2010, ApJ, 722, 1162

Mazzali, P. A., Cappellaro, E., Danziger, I. J., Turatto, M., \& Benetti, S. 1998, ApJL, 499, L49

McClelland, C. M., Garnavich, P. M., Galbany, L., et al. 2010, ApJ, 720, 704

Meyer, M. J., Zwaan, M. A., Webster, R. L., et al. 2004, MNRAS, 350, 1195

Miller, J. S., \& Stone, R. P. S. 1993, Lick Obs. Tech. Rep. 66 (Santa Cruz: Lick Obs.)

Milne, P. A., The, L.-S., \& Leising, M. D. 1999, ApJS, 124, 503

Moriya, T., Tominaga, N., Tanaka, M., et al. 2010, ApJ, 719, 1445

Mould, J. R., Hughes, S. M. G., Stetson, P. B., et al. 2000, ApJ, 528, 655

Narayan, G., Foley, R. J., Berger, E., et al. 2011, ApJL, 731, L11
Oke, J. B., Cohen, J. G., Carr, M., et al. 1995, PASP, 107, 375

Parrent, J. T., Thomas, R. C., Fesen, R. A., et al. 2011, ApJ, 732, 30

Pastorello, A., Valenti, S., Zampieri, L., et al. 2009, MNRAS, 394, 2266

Paturel, G., Petit, C., Prugniel, P., et al. 2003, A\&A, 412, 45

Perets, H. B., Gal-Yam, A., Mazzali, P. A., et al. 2010, Natur, 465, 322

Perlmutter, S., Aldering, G., Goldhaber, G., et al. 1999, ApJ, 517, 565

Phillips, M. M. 1993, ApJL, 413, L105

Phillips, M. M., Li, W., Frieman, J. A., et al. 2007, PASP, 119, 360

Phillips, M. M., Wells, L. A., Suntzeff, N. B., et al. 1992, AJ, 103, 1632

Poznanski, D., Chornock, R., Nugent, P. E., et al. 2010, Sci, 327, 58

Riess, A. G., Filippenko, A. V., Challis, P., et al. 1998, AJ, 116, 1009

Röpke, F. K. 2005, A\&A, 432, 969

Röpke, F. K. 2008, arXiv:0804.2147

Röpke, F. K., Woosley, S. E., \& Hillebrandt, W. 2007, ApJ, 660, 1344

Sahu, D. K., Tanaka, M., Anupama, G. C., et al. 2008, ApJ, 680, 580

Sako, M., Bassett, B., Becker, A., et al. 2008, AJ, 135, 348

Scalzo, R. A., Aldering, G., Antilogus, P., et al. 2010, ApJ, 713, 1073

Seitenzahl, I. R., Ciaraldi-Schoolmann, F., Röpke, F. K., et al. 2013, MNRAS, 429, 1156

Silverman, J. M., \& Filippenko, A. V. 2012, MNRAS, 425, 1917

Silverman, J. M., Foley, R. J., Filippenko, A. V., et al. 2012, MNRAS, 425, 1789

Silverman, J. M., Ganeshalingam, M., \& Filippenko, A. V. 2013, MNRAS, 430, 1030

Silverman, J. M., Ganeshalingam, M., Li, W., et al. 2011, MNRAS, 410, 585

Sirianni, M., Jee, M. J., Benítez, N., et al. 2005, PASP, 117, 1049

Sollerman, J., Lindahl, J., Kozma, C., et al. 2004, A\&A, 428, 555

Stanishev, V., Goobar, A., Benetti, S., et al. 2007a, A\&A, 469, 645

Stanishev, V., Taubenberger, S., Blanc, G., et al. 2007b, in AIP Conf. Proc. 924, The Multicolored Landscape of Compact Objects and Their Explosive Origins, ed. T. di Salvo, G. L. Israel, L. Piersant, L. Burderi, G. Matt, A. Tornambe, \& M. T. Menna (Melville, NY: AIP), 336

Sullivan, M., Guy, J., Conley, A., et al. 2011, ApJ, 737, 102

Taubenberger, S., Benetti, S., Childress, M., et al. 2011, MNRAS, 412, 2735

Taubenberger, S., Kromer, M., Pakmor, R., et al. 2013, ApJL, 775, L43

Theureau, G., Bottinelli, L., Coudreau-Durand, N., et al. 1998, A\&AS, 130, 333

Thomas, R. C., Aldering, G., Antilogus, P., et al. 2007, ApJL, 654, L53

Thomas, R. C., Aldering, G., Antilogus, P., et al. 2011a, ApJ, 743, 27

Thomas, R. C., Nugent, P. E., \& Meza, J. C. 2011b, PASP, 123, 237

Valenti, S., Pastorello, A., Cappellaro, E., et al. 2009, Natur, 459, 674

Valenti, S., Yuan, F., Taubenberger, S., et al. 2014, MNRAS, 437, 1519

Waldman, R., Sauer, D., Livne, E., et al. 2011, ApJ, 738, 21

Wang, X., Wang, L., Filippenko, A. V., Zhang, T., \& Zhao, X. 2013, Sci, 340,170

Wong, M. H., Pavlovsky, C., Long, K., et al. 2010, Wide Field Camera 3 Instrument Handbook, Version 2.0 (Baltimore, MD: STScI)

Wong, O. I., Ryan-Weber, E. V., Garcia-Appadoo, D. A., et al. 2006, MNRAS, 371,1855

Wood-Vasey, W. M., Miknaitis, G., Stubbs, C. W., et al. 2007, ApJ, 666, 694 